\title{
A Numerical Classification of the Genus Bacillus
}

\author{
By FERGUS G. PRIEST, ${ }^{*}$ MICHAEL GOODFELLOW ${ }^{2}$ AND \\ CAROLE TODD ${ }^{2}$ \\ ${ }^{1}$ Department of Brewing and Biological Sciences, Heriot-Watt University, \\ Edinburgh EHI $1 \mathrm{HX}, \mathrm{UK}$ \\ ${ }^{2}$ Department of Microbiology, The Medical School, Framlington Place, \\ Newcastle upon Tyne NE2 $4 \mathrm{HH}, \mathrm{UK}$
}

(Received 2 November 1987; revised 24 February 1988)

\begin{abstract}
Three hundred and sixty-eight strains of aerobic, endospore-forming bacteria which included type and reference cultures of Bacillus and environmental isolates were studied. Overall similarities of these strains for 118 unit characters were determined by the $S_{\mathrm{SM}}, S_{\mathrm{J}}$ and $D_{\mathrm{P}}$ coefficients and clustering achieved using the UPGMA algorithm. Test error was within acceptable limits. Six cluster-groups were defined at $70 \% S_{\mathrm{SM}}$, which corresponded to $69 \% S_{\mathrm{P}}$ and $48-57 \% S_{\mathrm{J}}$. Groupings obtained with the three coefficients were generally similar but there were some changes in the definition and membership of cluster-groups and clusters, particularly with the $S_{\mathrm{J}}$ coefficient.

The Bacillus strains were distributed among 31 major ( 4 or more strains), 18 minor ( 2 or 3 strains) and 30 single-member clusters at the $83 \% S_{\mathrm{SM}}$ level. Most of these clusters can be regarded as taxospecies. The heterogeneity of several species, including Bacillus brevis, $B$. circulans, B. coagulans, B. megateriun, B. sphaericus and B. stearothermophilus, has been indicated and the species status of several taxa of hitherto uncertain validity confirmed. Thus on the basis of the numerical phenetic and appropriate (published) molecular genetic data, it is proposed that the following names be recognized; Bacillus flexus (Batchelor) nom. rev., Bacillus fusiformis (Smith et al.) comb. nov., Bacillus kaustophilus (Prickett) nom. rev., Bacillus psychrosaccharolyticus (Larkin \& Stokes) nom. rev. and Bacillus simplex (Gottheil) nom. rev. Other phenetically well-defined taxospecies included ' $B$. aneurinolyticus', ' $B$. apiarius', ' $B$. cascainensis', ' $B$. thiaminolyticus' and three clusters of environmental isolates related to $B$. firmus and previously described as ' $B$. firmus $-B$. lentus intermediates'. Future developments in the light of the numerical phenetic data are discussed.
\end{abstract}

\section{INTRODUCTION}

Bacteria that produce heat-resistant endospores are classified in several genera in the family Bacillaceae. With the exception of the anaerobic, endospore-forming bacteria, the genus Bacillus is the largest and best-known member of this family, which also includes the genera Sporosarcina and Sporolactobacillus (Berkeley \& Goodfellow, 1981). Since endospore-formation is a universal feature of these bacteria, spore morphology has traditionally been given considerable weight in their classification and identification.

The earlier taxonomy of the bacilli was very confused, yielding more than 150 named species, often described on the basis of single physiological or ecological features. In a comparative study of over 1000 strains, Smith et al. (1952) used spore shape, size and location within the sporangium as a means of differentiating groups within the genus and reduced the number of species to 19. These morphological divisions have remained in general use (Wolf \& Barker, 1968; Hobbs \& Cross, 1983), despite criticism (Gordon, 1981). Revised and supplemented descriptions of common Bacillus species have also been published, together with information on 
some unclassified strains (Gordon et al., 1973). However, it was appreciated that the criteria used for this classification were insufficient (Gordon, 1981) and that many strains could not be accommodated within it. Nevertheless, the descriptions of Gordon and her co-workers form the basis of the classification in Bergey's Manual of Systematic Bacteriology (Claus \& Berkeley, 1986) and, together with strain histories, provide an invaluable framework for Bacillus taxonomists.

The inadequacy of Bacillus classification has been emphasized by molecular studies. The wide range of base composition in chromosomal DNA indicates genetic diversity (Priest, 1981; Fahmy et al., 1985) and suggests that Bacillus species should be reclassified into several genera. Analysis of rRNA by partial oligonucleotide sequencing has indicated a close relationship between the genera Bacillus, Planococcus, Sporosarcina, Staphylococcus and Thermoactinomyces and revealed Bacillus as a fairly coherent taxon (Stackebrandt \& Woese, 1981; Stackebrandt $e t$ al., 1987) equivalent in phylogenetic depth to the actinobacteria (Goodfellow \& Cross, 1984) or the enteric bacteria-vibrio group (Stackebrandt \& Woese, 1981), each of which encompasses several genera. Further, DNA homology studies have shown that many accepted Bacillus species, notably $B$. circulans (Nakamura \& Swezey, 1983a), B. megaterium (Hunger \& Claus, 1981), B. sphaericus (Krych et al., 1980) and B. stearothermophilus (Sharp et al., 1980), are markedly heterogeneous and in need of taxonomic revision.

Taxometric studies using a wide range of characters have been shown to be effective for the taxonomic revision of large groups of related bacteria (Goodfellow \& Dickinson, 1985; MacDonell \& Colwell, 1985). The extensive data bases derived from such studies are increasingly being used for the construction of probabilistic identification matrices (Williams $e t$ al., 1985) and for designing media formulations that are selective for the isolation of industrially important bacteria (Goodfellow \& Williams, 1986). Numerical taxonomy has been used to classify marine bacilli (Bonde, 1975; Boeyé \& Aerts, 1976), and culture collection strains representing the genus Bacillus have been analysed for a small number of classical tests (Priest $e t$ al., 1981). However, in a more comprehensive study Logan \& Berkeley (1981) concluded that further information was needed before Bacillus could be subdivided into 'three or more different genera', and 'spectra of strains', notably the $B$. firmus/B. lentus and B. circulans groups, be unscrambled. Although much remains to be done, these and other studies indicated the value of the numerical taxonomic approach in helping to clarify relationships within the genus Bacillus.

The primary aim of the current investigation was to establish the detailed intrageneric relationships of bacilli by examining representative strains for many properties using the numerical taxonomic procedure. It was also anticipated that the resultant data base would be used to construct a frequency matrix for the probabilistic identification of bacilli and for the formulation of media selective for specific bacilli of industrial importance.

\section{METHODS}

Strains and culture conditions. Three hundred and sixty-eight test strains were obtained from public and private collections (Table 1); 29 duplicate cultures were also included. Wherever possible type cultures were included. All cultures were stored on nutrient agar (Oxoid CM1) slopes at $4{ }^{\circ} \mathrm{C}$, with the inclusion of $5 \%(\mathrm{w} / \mathrm{v}) \mathrm{NaCl}$ for $B$. pantothenticus and adjusted to $\mathrm{pH} 6.0$ with $1.0 \mathrm{M}-\mathrm{HCl}$ for $B$. coagulans strains. Suspensions of vegetative cells and endospores were stored in glycerol $(20 \%, \mathrm{v} / \mathrm{v})$ at $-20^{\circ} \mathrm{C}$.

Each strain was examined for 118 unit characters (Tables 3-5). Thawed glycerol suspensions were used as inocula wherever possible but for sugar fermentation and organic acid utilization tests 2- to 4-d-old cultures grown on nutrient agar and suspended in physiological saline were used. All tests were done at least once on each strain but were repeated where ambiguous or clearly unexpected results were obtained. Inoculated media were usually incubated at $30^{\circ} \mathrm{C}$ but thermophilic and psychrophilic strains were incubated at $50{ }^{\circ} \mathrm{C}$ and $15{ }^{\circ} \mathrm{C}$, respectively. Morphological, degradation (with the exception of aesculin, allantoin, arbutin, hippurate and urea, which were done in test tubes), antibiotic sensitivity and physiological tests were done in Petri dishes. Replidishes (Sterilin) were used for 'spreading' organisms such as $B$. alvei and $B$. mycoides. They were also used for sugar fermentation and organic acid utilization tests. Petri and Replidishes were inoculated with a multipoint inoculator (Denley).

Morphology and pigmentation. Colonial morphology was examined on isolated colonies grown on nutrient agar for 2-4 d. Cellular morphology was examined in Gram-stained smears of these cultures, and spores were stained using malachite green (Cowan, 1974). Spore morphology was examined on cultures from soil-extract agar (SxA) (Gordon et al., 1973) in cases where sporulation did not occur on nutrient agar (see Tables 3-5). 
Degradative tests. The degradation of adenine and tyrosine $(0 \cdot 5 \%)$, elastin $(0 \cdot 3 \%)$, casein $(1 \%$, w/v, skimmed milk), guanine $(0.05 \%)$ and testosterone $(0.1 \%)$ was determined in nutrient agar after 7 and $14 \mathrm{~d}\left(2\right.$ and $5 \mathrm{~d}$ at $50{ }^{\circ} \mathrm{C}$ for thermophiles; 14 and $21 \mathrm{~d}$ at $15^{\circ} \mathrm{C}$ for psychrophiles); clearing of the areas under and around the growth was scored as positive. Gelatin $(0.4 \%)$ and $\operatorname{starch}(1 \%)$ hydrolysis were detected in the same basal medium after $7 \mathrm{~d}(2 \mathrm{~d}$ for thermophiles; $14 \mathrm{~d}$ for psychrophiles) by flooding plates with acidified $\mathrm{HgCl}_{2}$ (Frazier, 1926) and iodine solution (Gordon et al., 1973) respectively. Hydrolysis of DNA $(0.2 \%)$ and RNA $(0.3 \%)$ was observed using Bacto DNase Test agar (Difco) and nutrient agar as nutrient bases, respectively. After incubation for $7 \mathrm{~d}(2 \mathrm{~d}$ for thermophiles; $14 \mathrm{~d}$ for psychrophiles) plates were flooded with $1 \mathrm{M}-\mathrm{HCl}$ and clear zones recorded as positive. Tweens 20 and $80(1 \%, v / v)$ were incorporated into Sierra's (1957) medium and plates examined for opacity after $7 \mathrm{~d}$ ( $2 \mathrm{~d}$ for thermophiles; $14 \mathrm{~d}$ for psychrophiles). The hydrolysis of allantoin and urea was detected using the media and methods of Gordon $(1966,1968)$. Aesculin and arbutin (both $0 \cdot 1 \%$ ) degradation was determined by the methods of Williams et al. (1983) and examined after $7 \mathrm{~d}$ ( $2 \mathrm{~d}$ for thermophiles; $14 \mathrm{~d}$ for psychrophiles). Pullulan and pustulan hydrolysis was determined by the methods of Morgan et al. (1979) and Martin et al. (1980), respectively. Chitinolytic activity was observed after 14 and $21 \mathrm{~d}(3$ and $5 \mathrm{~d}$ for thermophiles) as the appearance of zones of clearing in colloidal chitin agar (Hsu \& Lockwood, 1975) and hippurate hydrolysis using the method of Gordon et al. (1973) after incubation for $14 \mathrm{~d}$ ( $5 \mathrm{~d}$ for thermophiles). Lecithinase activity was determined as opalescence in a medium comprising egg-yolk emulsion $(5 \%, \mathrm{v} / \mathrm{v} ;$ Oxoid) in nutrient agar incubated for $2 \mathrm{~d}(1 \mathrm{~d}$ for thermophiles; $5 \mathrm{~d}$ for psychrophiles). Pectin degradation was detected using the modified method of Williams et al. (1983); hydrolysis zones were detected after $7 \mathrm{~d}$ ( $2 \mathrm{~d}$ for thermophiles; $14 \mathrm{~d}$ for psychrophiles).

Antibiotic resistance. Strains were examined for the ability to grow in nutrient agar supplemented with antibiotics (Sigma) at two concentrations (Table 3). The antibiotics used were benzylpenicillin, chloramphenicol, D-cycloserine, erythromycin, gramicidin, nalidixic acid, polymyxin sulphate, rifampicin, streptomycin sulphate and tetracycline. Growth was recorded after $7 \mathrm{~d}$ ( $3 \mathrm{~d}$ for thermophiles; $14 \mathrm{~d}$ for psychrophiles) and resistance scored as positive.

Acid production from sugars and sugar alcohols. This was detected using the media and methods of Gordon et al. (1973). Replidishes were inoculated and examined after $7 \mathrm{~d}$ ( $3 \mathrm{~d}$ for thermophiles; $14 \mathrm{~d}$ for psychrophiles) for acid production.

Organic acid utilization. The ability of strains to use organic acids was determined using the methods of Gordon et al. (1973). Replidishes were examined after $5 \mathrm{~d}$ ( $2 \mathrm{~d}$ for thermophiles; $10 \mathrm{~d}$ for psychrophiles) for the appropriate colour change.

Tolerance tests. Nutrient agar was used as the basal medium. Growth at $5{ }^{\circ} \mathrm{C}$ and $17{ }^{\circ} \mathrm{C}$ was recorded after 14 and $21 \mathrm{~d}$, growth at $37^{\circ} \mathrm{C}$ after $3 \mathrm{~d}$, and growth at $50^{\circ} \mathrm{C}$ and $65^{\circ} \mathrm{C}$ after $2 \mathrm{~d}$. Growth at $\mathrm{pH} 4 \cdot 5,6 \cdot 0,8 \cdot 0$ and $9 \cdot 5$ was determined in media adjusted to the appropriate $\mathrm{pH}$ with $\mathrm{HCl}$ or $\mathrm{NaOH}$ and recorded after $7 \mathrm{~d}\left(3 \mathrm{~d}\right.$ at $\left.50^{\circ} \mathrm{C}\right)$. Growth in the presence of $\mathrm{NaCl}(2,5$ and $10 \%, w / v)$ was recorded after $7 \mathrm{~d}\left(3 \mathrm{~d}\right.$ at $\left.50^{\circ} \mathrm{C}\right)$.

Miscellaneous biochemical tests. Anaerobic growth was determined according to Gordon et al. (1973) and gas production from glucose in glucose/peptone water containing Durham tubes. Production of dihydroxyacetone and indole, reduction of nitrate, deamination of phenylalanine, and the Voges-Proskauer test were determined using the standard methods for Bacillus strains (Gordon et al., 1973). Hydrolysis of $o$-nitrophenyl $\beta$-D-galactoside, the methyl red test, the oxidase reaction and presence of phosphatase were examined using the procedures of Cowan (1974). Ability to grow on MacConkey agar (Oxoid) was recorded after $5 \mathrm{~d}\left(2 \mathrm{~d}\right.$ at $50^{\circ} \mathrm{C} ; 10 \mathrm{~d}$ at $15^{\circ} \mathrm{C}$ ).

Coding of data. Nearly all the characters existed in one of two mutually exclusive states and were scored plus (1) or minus (0). Qualitative multistate characters were each scored plus (1) for the character state shown and minus (0) for the alternatives. Quantitative multistate characters such as tolerance to $\mathrm{NaCl}$ were coded using the additive method of Sneath \& Sokal (1973). Characters which did not show any separation value or were poorly reproducible were deleted from the data matrix. The final $n \times t$ table, therefore, contained data for 368 bacteria $(t)$ and 118 unit characters ( $n$; Tables $3-5)$.

Computer analysis. Data were analysed using the Clustan 1C package (Wishart, 1978) on a Burroughs B6370 computer using the simple matching $\left(S_{\mathrm{SM}}\right)$, Jaccard $\left(S_{\mathrm{J}}\right)$ and pattern difference $\left(D_{\mathrm{P}}\right)$ coefficients (Sneath \& Sokal, 1973). Clustering was achieved using the unweighted pair group method with arithmetic averages (UPGMA) algorithm (Sneath \& Sokal, 1973).

Test reproducibility. Twenty-nine strains were tested in duplicate and an estimate of test variance calculated (formula 15; Sneath \& Johnson, 1972) which was used to calculate the average probability ( $p$ ) of an erroneous test result (formula 4; Sneath \& Johnson, 1972).

\section{RESULTS}

Test error

Experimental test error was calculated from the data collected on the 29 duplicate strains. The average probability $(p)$ of an erroneous test result was $3.90 \%$ calculated from the pooled 
variance $\left(S^{2}=0.0374\right)$ of all the unit characters for the duplicate cultures. The 29 pairs of duplicate strains showed a mean observed similarity of $93.86 \% S_{\mathrm{SM}}$. Some groups of tests were highly reliable, particularly cellular morphology, degradation, acid from sugars, growth, and miscellaneous tests, all of which displayed a variance $<0.03$. The most irreproducible tests were those involving organic acid utilization, in which the indicator change was difficult to read. Nevertheless, these results were included in the study because the variance $(0.113)$ was only slightly greater than the generally accepted level of $<0 \cdot 1$ (Sneath \& Johnson, 1972).

\section{Gross taxonomic structure}

The data were analysed using the $S_{\mathrm{SM}}, S_{\mathrm{J}}$ and $D_{\mathrm{P}}$ coefficients with the UPGMA algorithm. The $S_{\mathrm{SM}}$ dendrogram was divided into six aggregate clusters at the $70 \%$ similarity $(S$-) level (Fig. 1; Table 1), which corresponded to $69 \% S_{\mathrm{P}}$. The composition of the cluster-groups was slightly different in the $S_{\mathrm{SM}}$ and $D_{\mathrm{P}}$ phenograms (Table 2) but the major and minor clusters were little affected. In the $S_{\mathrm{J}} /$ UPGMA analysis, five cluster-groups were apparent but to delineate them a staggered line from 48 to $57 \%$ similarity was required. Given this relaxation of the generally accepted interpretation of dendograms, the composition of the cluster-groups showed good congruence with those obtained in the $S_{\mathrm{SM}}$ and $D_{\mathrm{P}}$ analyses. The major variation was observed in the distribution of the clusters of obligate aerobic strains within cluster-groups D and E. The $S_{\mathrm{SM}} /$ UPGMA analysis most closely resembled classifications obtained in earlier studies of the genus (Logan \& Berkeley, 1981; Priest et al., 1981) and it is presented here in detail.

The composition of cluster-group A was largely unaffected by the coefficients used (Table 2). The bacteria encompassed by this taxon all produced acid from a wide range of carbohydrates, were facultative anaerobes with ellipsoidal spores that distended the sporangium, and hydrolysed a variety of polysaccharides including starch and pullulan. Similarly, cluster-group B encompassed bacteria that were aerobic or facultatively anaerobic and produced acid from a variety of sugars. They also formed oval spores which, with the exception of those of $B$. laterosporus and 'B. psychrosaccharolyticus', did not distend the sporangium. Strains assigned to cluster-group B hydrolysed casein and, with the exception of $B$. pumilus, starch.

Cluster-group $C$ was based on $B$. firmus, $B$. pantothenticus, marine strains and perhaps $B$. lentus, although in the $S_{\mathrm{SM}} / \mathrm{UPGMA}$ and $D_{\mathrm{P}} / \mathrm{UPGMA}$ analyses this species was given clustergroup status. These bacteria were generally weak in their ability to form acid from sugars and grew poorly, if at all, under anaerobic conditions. They produced oval spores and were $\mathrm{NaCl}$ tolerant. Considerable affinity was found between cluster-groups $C$ and $D$, which included ' $B$. aneurinolyticus' and $B$. sphaericus, but strains in the latter group were distinguished by lack of acid production from sugars ( $B$. psychrophilus was a very weak acid-former). These bacteria displayed a variety of spore morphologies.

Cluster-group E contained B. lentus and B. macquariensis but the weight of evidence (Table 2) suggests that these taxa might more appropriately be placed in cluster-groups $D$ and $A$, respectively. Cluster-group $F$ encompassed the two thermophilic taxa $B$. coagulans and $B$. stearothermophilus. These bacteria displayed heterogeneity of spore morphology and fermented a variety of carbohydrates.

The full characteristics of the cluster-groups are given in Table 3.

\section{Composition and characteristics of major and minor clusters}

The strains were recovered in 31 major (four or more strains), 18 minor (two or three strains) and 30 single-member clusters at the $83 \% S_{\mathrm{SM}}$ level (Fig. 1). These clusters have been assigned names according to the distribution of type and reference strains. The characteristics of the major and minor clusters are given in Tables 4 and 5, respectively.

Within cluster-group A, cluster 1 contained 13 strains received as $B$. alvei. They formed a homogeneous phenon at $87 \% S_{\mathrm{SM}}$ and displayed typical motile micro-colonies (see Parry et al., 1983) and swollen sporangia containing oval, terminal spores. Cluster 3 comprised four strains of ' $B$. thiaminolyticus' that were morphologically similar to $B$. alvei but distinguishable by nonmotile micro-colonies and positive and negative reactions in the nitrate reduction and Voges- 
Proskauer tests, respectively. Of the six strains assigned to cluster 4, four were originally labelled as $B$. circulans, one as $B$. alvei and the other as ' $B$. sphaericus var. rotans'. These bacteria possessed motile micro-colonies typical of $B$. alvei but differed from the latter in failing to produce dihydroxyacetone and in being negative for nitrate reduction and the Voges-Proskauer reaction. Cluster 7 strains resemble $B$. pabuli (Nakamura, 1984 $a$ ) and were named accordingly.

The ten strains of $B$. macerans recovered in cluster 5 displayed the typical reactions of this species, in particular the production of gas from sugars, a property shared with $B$. polymyxa (cluster 8 ). However, the strains in the latter taxon fermented a less extensive range of sugars, hydrolysed casein and produced dihydroxyacetone. Related to $B$. polymyxa at $77.5 \% S_{\mathrm{SM}}$ were five strains of $B$. circulans including the type strain (cluster 6 ). These bacteria did not produce gas from glucose. The heterogeneity of strains received as $B$. circulans was evident given their assignment to two major, three minor and four single-member clusters. The sole strain of ' $B$. filicolonicus' was recovered as a single member cluster in cluster-group A.

Cluster-group B was numerically the largest in the study. Strains of B. cereus, B. mycoides and $B$. thuringiensis, assigned to cluster 11 within this cluster-group, were divided at the 89 to $92 \%$ $S_{\mathrm{SM}}$ level into nine subclusters which approximated to the species and varieties represented. Subclusters $11 \mathrm{~A}$ and $11 \mathrm{~B}$ were heterogeneous and contained strains labelled $B$. thuringiensis and $B$. cereus. Subcluster $11 \mathrm{C}$ contained seven strains of $B$. cereus, some of which had been associated with food poisoning. Subcluster $11 \mathrm{D}$ also contained $B$. cereus strains, some of which were originally designated ' $B$. cereus var. fluorescens' and ' $B$. cereus var. albolactis'. $B$. thuringiensis strains were recovered in subcluster $11 \mathrm{E}$ and two $B$. cereus strains of serotypes 6 and 8 comprised $11 \mathrm{~F}$. Twelve strains of $B$. thuringiensis, including the type strain, formed subcluster $11 \mathrm{G}$. Subcluster $11 \mathrm{H}$ was largely composed of $B$. cereus strains, and the final subcluster 11I, contained four strains of B. mycoides. Bacillus cereus NCIB 8705 and a marine isolate representative of cluster IIC (B. cereus) of Bonde (1975) formed single-member subclusters. Although the subclusters largely conformed to the designations $B$. cereus, $B$. mycoides and $B$. thuringiensis, consistent features that distinguished them, with the exception of the rhizoidal colony forms of $B$. mycoides, were not evident. Loosely associated with the $B$. cereus cluster were two marine isolates from group IIC of Bonde (1975), and two strains of ' $B$. psychrosaccharolyticus'.

Eight strains of $B$. laterosporus, including the type strain, were recovered in cluster 13 . Their close affinity to $B$. cereus $\left(76 \% S_{\mathrm{SM}}\right)$ may initially seem surprising, but if the unusual spore morphology is ignored, the taxa have many features in common. Both species contained facultative anaerobes that were largely methyl red positive and reduced nitrate; both degraded a variety of macromolecules and produced acid from a similar range of sugars. A single strain of ' $B$. pycnoticus' recovered within the $B$. laterosporus cluster at $86 \% S_{\mathrm{SM}}$ did not have the characteristic lateral spore position of $B$. laterosporus.

The ' $B$. subtilis group', including $B$. megaterium, joined $B$. cereus at $72 \% S_{\mathrm{SM}}$. Cluster 14 contained nine strains of which eight were authentic cultures of $B$. amyloliquefaciens or were strains labelled $B$. subtilis from amylase fermentations; one strain was a marine isolate. Although cluster 14 was distinct from $B$. subtilis (cluster 15), consistent differential features were not evident. Fermentation of meso-inositol, lactose and xylose, and hydrolysis of DNA and Tween 80 provide some measure of distinction.

Cluster 15 encompassed strains received as $B$. subtilis, including the type strain. Two strains received as ' $B$. vulgatus' and two designated as ' $B$. aterrimus' were recovered in this cluster. Two marine isolates, representatives of group IVA $(B$. subtilis) and group IIB ( $B$. megaterium) of Bonde (1975), were assigned to this cluster as was a second ' $B$. pycnoticus' strain. Bacteria in cluster 15 conformed to the typical description of $B$. subtilis since they were obligate aerobes that were positive in the nitrate reduction and Voges-Proskauer tests and produced acid from a variety of sugars.

Strains of $B$. pumilus formed a homogeneous cluster related to B. subtilis at $79 \% S_{\mathrm{SM}}$. Most of these organisms were received as $B$. pumilis, including two marine isolates, representing Bonde's (1975) group IVB (B. pumilus). However, representatives of his group IIB (B. megaterium) and 


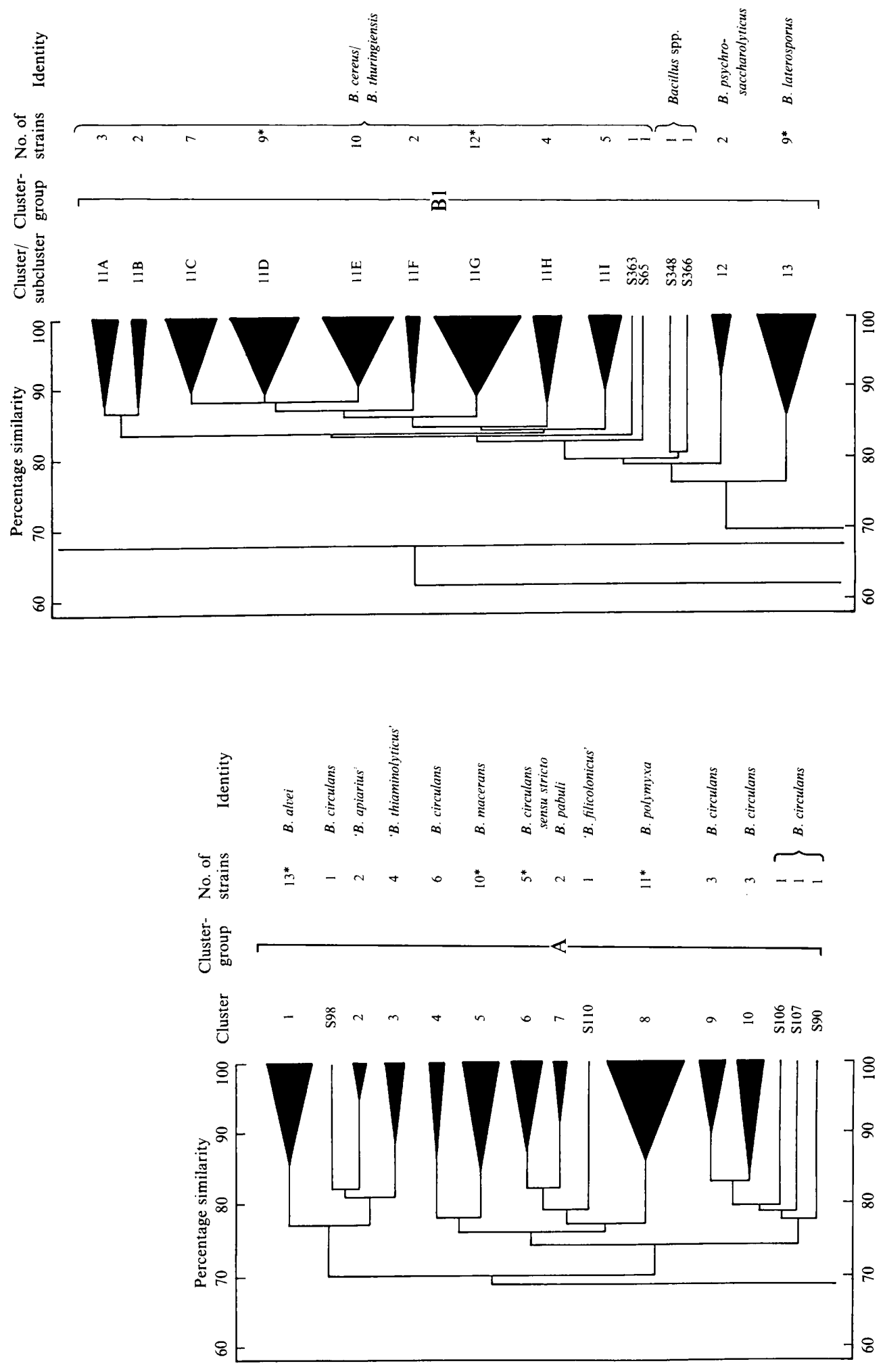




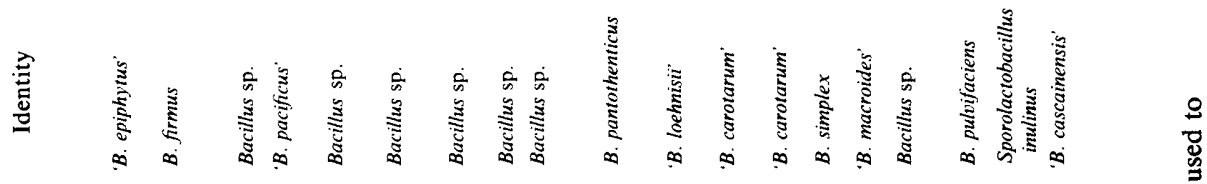

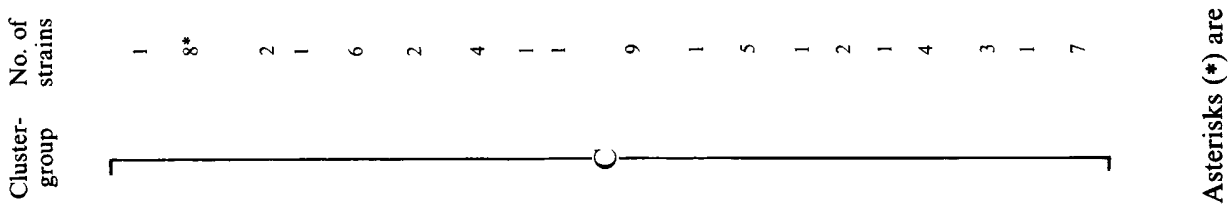

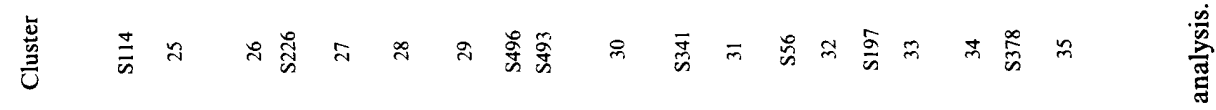

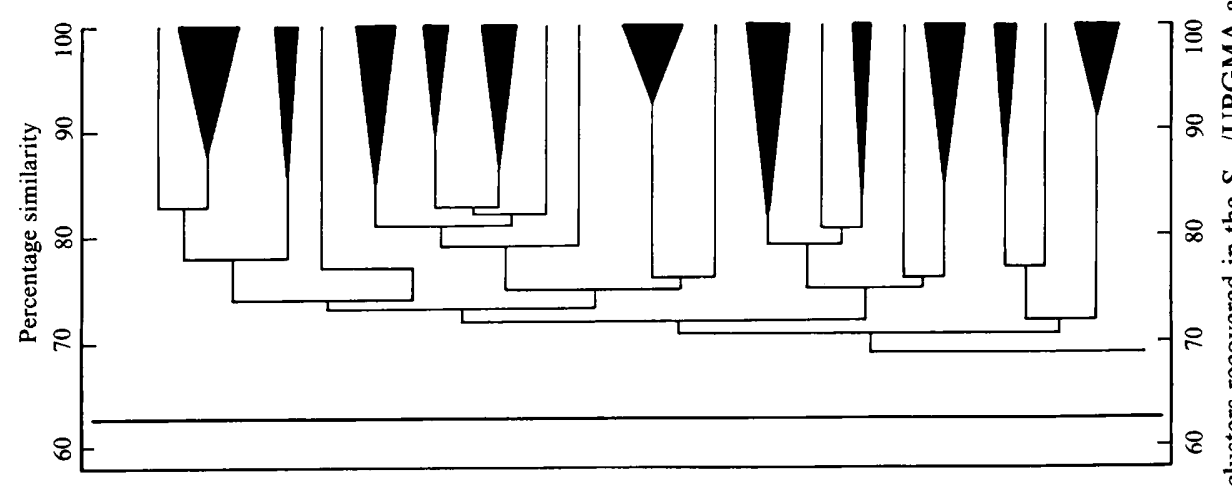

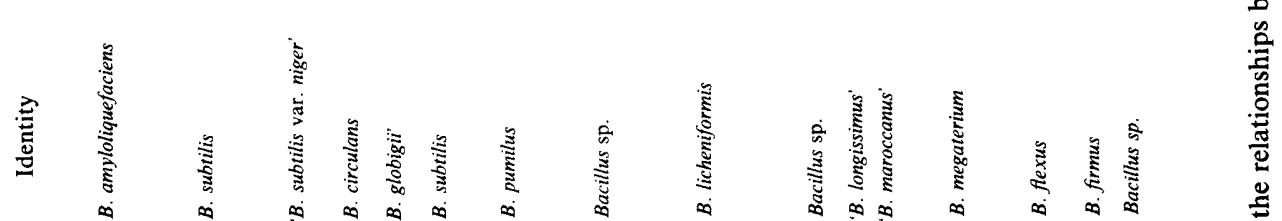

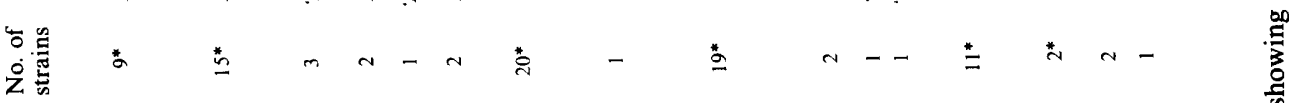
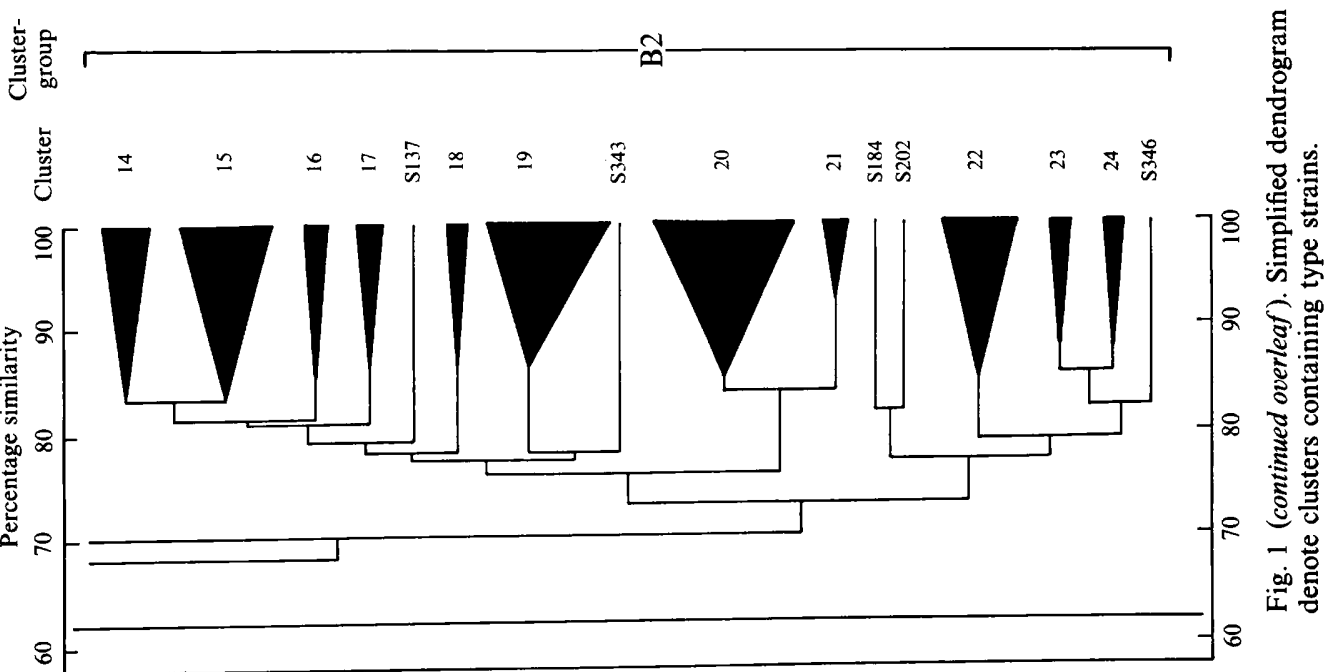


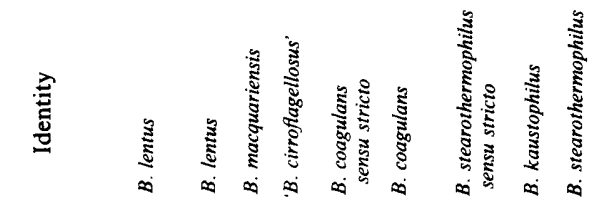

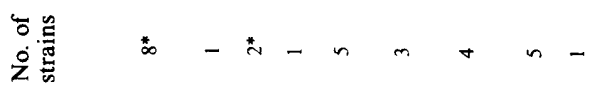

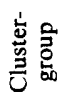

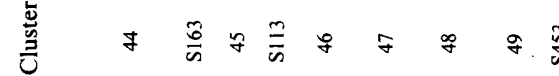

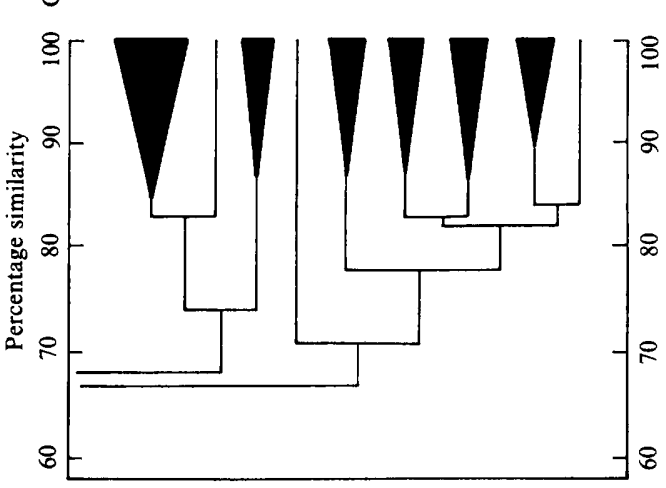

要

吾

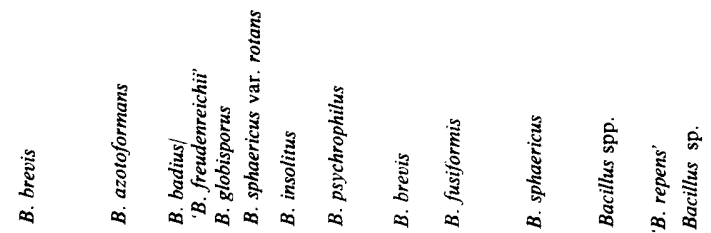

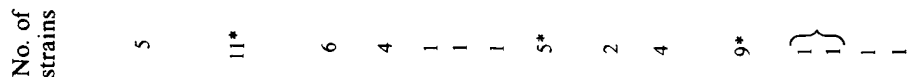

产言

$\frac{5}{2}$

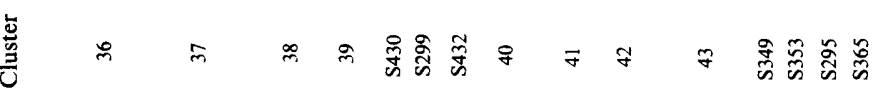

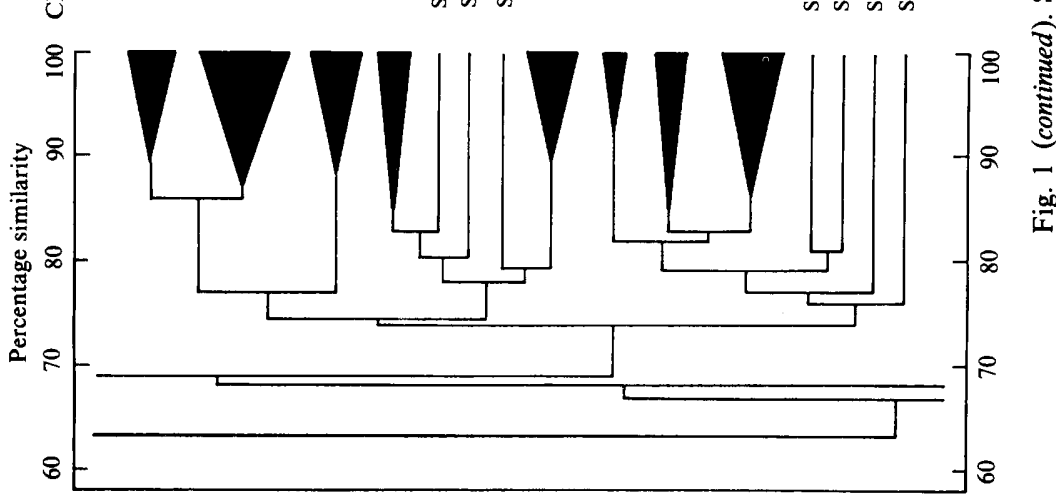


Table 1. Designation and source of strains assigned to cluster-groups (defined at $70 \% S_{\mathrm{SM}}$, $U P G M A$ ) and clusters (defined at $83 \% S_{\mathrm{SM}}, U P G M A$ )

Binomials in inverted commas are not on the Approved Lists of Bacterial Names (Skerman et al., 1980) and have not been validly published since 1 January 1980 . Type strains are marked with an asterisk (*).

Cluster-group A

Strains assigned to cluster 1 (Bacillus alvei)

S3-*S5

S6-S8

S9

S11-S16

S414, S415

S327-S330

S10

S93, S94, S96, S103

S310

S185

S186-S189, *S191

S192

S193-S195

S89, S91

*S92, S95

S109

S101, S102

S247, S249, S280

*S251

S254-S256, S258-S261

S88

S97, S104

S99, S100, S105

S90

S98

S106

S107

S110
B. alvei, NCIB 8212, NCIB 8199, NCIB 9371

$B$. alvei, NCTC 3324, NCTC 3349, NCTC 7583

B. alvei, J. R. Norris, Cadbury Schweppes Ltd, Reading, UK, BO 113

B. alvei, WR 2772, WR 2773, WR 3186 (E. Schreiner, SK G), WR 3187 (E. Schreiner, 4N), WR 3250 (E. Schreiner, A), WR 3251 (E. Schreiner, B)

Strains assigned to cluster 2 ('Bacillus apiarius')

'B. apiarius', R. E. Gordon, Rutgers University, New Jersey, USA, NRS 1438 (H. Katznelson, BX3), NRS 1439 (H. Katznelson, BX5); bee larvae

Strains assigned to cluster 3 ('Bacillus thiaminolyticus')

'B. thiaminolyticus', J. R. Norris, BO 286-BO 289 (J. Yamaguchi, M1-M4)

Strains assigned to cluster 4 (Bacillus circulans)

B. alvei, J. R, Norris, BO 024

B. circulans, J. R. Norris, BO 030, BO 061, BO 197 (T. Gibson, 514), BO 319

'B. sphaericus var. rotans', H. J. Somerville, Shell, UK, T216

Strains assigned to cluster 5 (Bacillus macerans)

B. macerans, T. R. G. Gray, University of Essex, UK, NCIB 7588

B. macerans, NCIB 8160, NCIB 8210, NCIB 8930, NCIB 10443, NCIB 9368

B. macerans, H. J. Somerville, T521

B. macerans, WR 1013, WR 1014, WR 2614 (colonial variant of WR 1014)

Strains assigned to cluster 6 (Bacillus circulans sensu stricto)

B. circulans, P. A. Hartman, Iowa State University, Ames, USA, NRRL B-381, NRRL B-380

B. circulans, J. R. Norris, BO 004 (NCTC 2610), BO 196 (NCTC 5849)

B. circulans, NCIB 9555

Strains assigned to cluster 7 (Bacillus pabuli)

B. circulans, J. R. Norris, BO 317 (T. Gibson, 261), BO 318 (T. Gibson, 287)

Strains assigned to cluster 8 (Bacillus polymyxa)

B. polymyxa, P. A. Hartman, IA 32 (J. C. Ayres, B-57-3B), ATCC 8523, IA 56

B. polymyxa, NCIB 8158

B. polymyxa, WR 1417, WR 1756, WR 1966, WR 2161 (banana skin), WR 2179, WR 2186 (garden soil), WR 2494 (potato)

Strains assigned to cluster 9 (Bacillus circulans)

B. circulans, P. A. Hartman, NRRL B-378

B. circulans, J. R. Norris, BO 266 (NCTC 7578), BO 320 (T. Gibson, 48)

Strains assigned to cluster 10 (Bacillus circulans)

B. circulans, J. R. Norris, BO 305 (T. Gibson, 137), BO 306 (T. Gibson, 255), BO 321

(T. Gibson, 38)

Single-member clusters

B. circulans, P. A. Hartman, NRRL B-395

B. circulans, J. R. Norris, BO 267; NCTC 9432

B. circulans, J. R. Norris, BO 322; T. Gibson, 92

B. circulans, J. R. Norris, BO 323; T. Gibson, 279

'B. filicolonicus', J. R. Norris, BO 322; T. Gibson, 92 
Table 1 (continued)

Cluster-group B1

Strains assigned to cluster 11 (Bacillus cereus/Bacillus thuringiensis)

Subcluster 11A ('Bacillus thuringiensis var. finitimus')

S120

'B. finitimus', J. R. Norris, BO 308; T. Gibson, 1316

S332

'B. thuringiensis var. alesti', P. A. Hartman, BT-3 (serotype 3a)

S121

'B. thuringiensis var. finitimus', P. A. Hartman, BT-2

Subcluster 11B (Bacillus cereus)

$\begin{array}{ll}\text { S66 } & \text { B. cereus, T. R. G. Gray, B20; NCTC } 6474 \\ \text { S297 } & \text { 'B. sotto', J. R. Norris, BO 021 }\end{array}$

Subcluster 11C (Bacillus cereus)

S58

$\mathrm{S} 62, \mathrm{~S} 63$

S67

B. cereus, B. Austin, Heriot-Watt University, Edinburgh, UK, SA 15; swan faeces

$\mathrm{S} 68$

B. cereus, P. A. Hartman, IA 36 (Y. L. Quinn; ATCC 11778), NRRL B-344

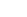

B. cereus, T. R. G. Gray, B21; NCTC 7464

S72

B. cereus, R. J. Gilbert, Central Public Health Laboratories, Colindale, London, UK, $3502 / 73$; fried rice (serotype 5)

S86

B. cereus, R. J. Gilbert, 4433/73; meat loaf

Subcluster 11D (Bacillus cereus)

*S60

S61, S76

S74

S75

S77

579

S81

S470

B. cereus var. terminalis', WR 7100

Subcluster 11E (Bacillus thuringiensis)

S64

S334

B. cereus, J. R. Norris, BO 002; DSM 31

B. cereus, P. A. Hartman, IA 27, NRS 996

B. cereus, R. J. Gilbert, $4746 / 77$; fried rice (serotype 1)

B. cereus, H. J. Somerville, TI 87; strain T

'B. cereus var. albolactis', NCIB 5097

'B. cereus var. fluorescens', H. J. Somerville, TI 53; NCIB 2600

'B. cereus var. mycoides', NCTC 2603

'B. thuringiensis var. dendrolinus', J. R. Norris, 10; H. Dulmage, 37 (serotype 4ab)

S476, S477

S471

S472

S466, S467

B. cereus, NCIB 6349

B. thuringiensis, WR 4138

'B. thuringiensis var. aizawai', J. R. Norris, 16 (H. Dulmage, 227), 17 (H. Dulmage, 137) (serotype 7)

S468

S478

'B. thuringiensis var. benyae', J. R. Norris, 11; H. Dulmage, 136 (serotype 4ac)

'B. thuringiensis var. galleriae', J. R. Norris, 12; H. Dulmage, 273 (serotype 5ab)

'B. thuringiensis var. kurstaki', J. R. Norris, 6 (H. Dulmage, 187), 7 (H. Dulmage, 89) (serotype $3 \mathrm{ab}$ )

'B. thuringiensis var. sotto', J. R. Norris, 8; H. Dulmage 5 (serotype 4ab)

Subcluster $11 \mathrm{~F}$ (Bacillus cereus)

S70, S71

B. cereus, R. J. Gilbert, 4370/75 (serotype 6; barbecued chicken), 4431/73 (serotype 8; Indonesian rice dish)

Subcluster 11G (Bacillus thuringiensis)
S335
B. thuringiensis, WR 5751
*S336
B. thuringiensis, T. R. G. Gray, B76; NCIB 9134
S337
S464, S465
B. thuringiensis, H. J. Somerville, T 537 (serotype 1)
S331
S469
S474
$\$ 463$
S473
S461, S462
'B. thuringiensis var. alesti', J. R. Norris, 4 (H. Dulmage, 10), 5 (H. Dulmage, 104)
'B. thuringiensis var. berliner', P. A. Hartman, BT-1; Bonnefoi, BT-1
'B. thuringiensis var. dendrolinus', J. R. Norris, 9; H. Dulmage, 106 (serotype 4b)
'B. thuringiensis var. entomocidus', J. R. Norris, 14; H. Dulmage, 9 (serotype 6)
'B. thuringiensis var. finitimus', J. R. Norris, 3; H. Dulmage, 3
'B. thuringiensis var. galleriae', J. R. Norris, 13; H. Dulmage, 29
'B. thuringiensis var. thuringiensis', J. R. Norris, 1 (H. Dulmage, 39), 2 (H. Dulmage, 17) (serotype 7)
Subcluster $11 \mathrm{H}$ (Bacillus cereus/Bacillus thuringiensis)
S69, S73
S78
B. cereus, R. J. Gilbert, 3605/73 (serotype 3; boiled rice), 4810/72 (serotype 1; vomit)
S475
'B. cereus subsp. albolactis', NCIB 8079
B. thuringiensis, J. R. Norris, 15

Subcluster 11 ('Bacillus cereus var. mycoides')

S80

S83-S85

'B. cereus var. mycoides', NCIB 926

S482

'B. cereus var. mycoides', WR 1541, WR 2500, WR 2528

'B. cereus var. mycoides', H. J. Somerville, T193 
S438, S439

S145-S148, S150

S151

*S152, S154

S289

S65

S348

S363

S366

*S18-S20

S21

S24

S23

S234

S312

S352

S30

S22

S290

S31, *S316, S317

S339, S340

S311, S315

S321, S322

S32

S359, S362

S223, S224

S225

S87

S351

S319

S323

S208, S273, S274

S209, S275, S276

S278, *S279

S281, S282

S283, S284

S285-S288

S354, S355, S358, S361
Table 1 (continued)

Strains assigned to cluster 12 (Bacillus psychrosaccharolyticus)

'B. psychrosaccharolyticus', J. L. Stokes, T25B, T27B; soil

Strains assigned to cluster 13 (Bacillus laterosporus)

B. laterosporus, J. R. Norris, BO 026, BO 115 (T. Gibson, 308), BO 116 (T. Gibson, 1066), BO 262, BO 309 (T. Gibson, 1080)

B. laterosporus, WR 2197

B. laterosporus, NCIB 8215, NCIB 11046

'B. pycnoticus', J. R. Norris, BO 311 (T. Gibson, 51)

Single-member clusters

B. cereus, NCIB 8705

Bacillus sp., G. J. Bonde, 1 (cluster IIC; B. cereus)

Bacillus sp., G. J. Bonde, 354 (cluster IIC; $B$. cereus)

Bacillus sp., G. J. Bonde, 372 (cluster IIC; B. cereus)

\section{Cluster-group B2}

Strains assigned to cluster 14 (Bacillus amyloliquefaciens)

B. amyloliquefaciens, F. E. Young, University of Rochester, NY, USA, F (L. L. Campbell, F), H (L. L. Campbell, H), K (L. L. Campbell, K)

B. amyloliquefaciens, NCIB 10785

B. amyloliquefaciens, J. R. Norris, 30; ATCC 23843

B. subtilis, J. R. Norris, 29, T. Kaneko, N

B. subtilis, ABM Chemical Ltd, Stockport, UK, B20; amylase fermentation

B. subtilis, P. A. Hartman, JR 8; J. Robyt, amylase preparation

Bacillus sp., G. J. Bonde, 50 (cluster 3A)

Strains assigned to cluster 15 (Bacillus subtilis)

'B. aterrimus', J. R. Norris, BO 096; T. Gibson, 525

B. megaterium, J. R. Norris, $25 ;$ T. Kaneko, 203

'B. pycnoticus', J. R. Norris, BO 322

B. subtilis, NCIB 2591, NCIB 3610, NCIB 8054

'B. vulgatus', NCIB 8063, NCIB 8802

B. subtilis, P. A. Hartman, IA 5, W 23

B. subtilis, J. R. Norris, 6 (T. Gibson, 1115), 7 (T. Gibson, 1137)

'B. aterrimus', WR 2192; NCIB 8055

Bacillus sp., G. J. Bonde, 177 (cluster 2B; B. megaterium), 315 (cluster 4A; B. subtilis)

Strains assigned to cluster 16 ('Bacillus subtilis var. niger')

'B. niger', J. R. Norris, BO 099 (T. Gibson, 1208), BO 098 (T. Gibson, 1007)

' $B$. subtilis var. niger', E. Hemphill, Syracuse University, NY, USA, 1000

Strains assigned to cluster 17 (Bacillus circulans)

B. circulans, P. A. Hartman, NRRL B-377

Bacillus sp., G. J. Bonde, 47 (cluster IIIA)

Strains assigned to cluster 18 (Bacillus subtilis)

B. subtilis, J. R. Norris, 2; T. Gibson, 636

B. subtilis, WR 2745

Strains assigned to cluster 19 (Bacillus pumilus)

B. megaterium, B. Austin, SA 217, SA 232, SA 218; swan faeces

B. megaterium, B. Austin, CGA 59, G-2-P, CGA 28; Canada goose faeces

B. pumilus, T. R. G. Gray, B46 (NCTC 7576), B47 (NCTC 8241)

B. pumilus, P. A. Hartman, NRRL B-3275, NRS 630

B. pumilus, NCTC 2595, NCTC 2596

B. pumilus, J. R. Norris, 10 (T. Gibson, 1130), 12 (T. Gibson, 10), 13 (T. Gibson, 47), 14 (T. Gibson, 67)

Bacillus sp., G. J. Bonde, 86 (cluster IV, B. pumilus), 88 (cluster IVB; B. pumilus), 174

(cluster IIB; B. megaterium), 293 (cluster 2C, B. cereus)

Strains assigned to cluster 20 (Bacillus licheniformis)

B. licheniformis, P. A. Hartman, 9945A; C. B. Thorne, 9945A 
*S168-S173

S174-S180

S181-S183

S314

S338

S356, S357

S205

S216-S218

S215

S219

S220-*S222

S296

S379

S211, S212

S412

S347

S137

S184

S202

S343

S346

\section{Table 1 (continued)}

B. licheniformis, J. R. Norris, 17 (DSM 13), 18 (T. Gibson, 1174), 20 (T. Gibson, 1174), 22 (T. Gibson, 1160), 23 (T. Gibson, 5), 24 (T. Gibson, 1158)

B. licheniformis, NCIB 6816, NCIB 7224, NCIB 8061, NCIB 8537, NCIB 8549, NCIB 8874, NCIB 9668

B. licheniformis, NCTC 962, NCTC 1097, NCTC 2120

B. subtilis, P. A. Hartman, R66-A

B. subtilis, NCIB 9536

Strains assigned to cluster 21 (Bacillus sp.)

Bacillus sp., G. J. Bonde, 127 (cluster V), 128 (cluster V)

Strains assigned to cluster 22 (Bacillus megaterium)

B. megaterium, B. Austin, SA 174; swan faeces

B. megaterium, J. R. Norris, BO 075 (T. Gibson, 386), BO 076, BO 077 (T. Gibson, 732)

B. megaterium, P. A. Hartman, NRRL B-348

B. megaterium, J. R. Norris, BO 078, T. Gibson, 186

B. megaterium, NCIB 7581, NCIB 8291, NCIB 9376

'B. silvaticus', NCIB 8674

'B. malabarensis', NCTC 5637

Strains assigned to cluster 23 (Bacillus flexus)

B. megaterium, R. E. Gordon, NRS 602 (J. R. Porter; G. Brederman; 'B. agrestis'), NRS 665 (B. S. Henry, 'B. flexus', 131)

Strains assigned to cluster 24 (Bacillus firmus)

B. firmus, WR 3389; R. E. Gordon, NRS 1147

Bacillus sp., A. Boeyé, Vrije Universiteit, Brussels, Belgium, VUB 231 (group B1); North Sea sediment

Single-member clusters

'B. globigii', P. A. Hartman, IA 30

'B. longissimus', J. R. Norris, BO 339

'B. maroccanus', NCIB 10500

Bacillus sp., A. Boeyé, VUB 72 (group A2); North Sea sediment

Bacillus sp., A. Boeyé, VUB 211 (group A1); North Sea sediment

\section{Cluster-group C}

Strains assigned to cluster 25 (Bacillus firmus)

S122, *S123

S126, S127, S129, S130, S131

$\mathrm{S} 132$

S413

S483

S342, S344

S497, S492, S487, S494

S482, S495

S484, S488-S490

S227, S228

$\mathrm{S} 230$

S231, S233-S237
B. firmus, NCIB 8162, NCIB 9366 (NRS 613)

B. firmus, WR 3317 (R. E. Gordon, NRS 858), WR 3318 (R. E. Gordon, NRS 861), WR 3320 (R. E. Gordon, NRS 860), WR 3384 (R. E. Gordon, NRS 1070), WR 3385 (R. E. Gordon, NRS 1131)

B. firmus, H. J. Somerville, T 544

Strains assigned to cluster 26 (Bacillus sp.)

B. firmus, WR 3390

Bacillus sp., NRS 1151

Strains assigned to cluster 27 (Bacillus sp.)

Bacillus sp., A. Boeyé, VUB 33 (cluster B4), VUB 73 (cluster B2); North Sea sediment Bacillus sp., R. E. Gordon, NRS 1574, NRS 1565, NRS 1570, NRS 1566; M. Turner, SM 34, SM 23, SM 29, SM24; salt marsh

Strains assigned to cluster 28 (Bacillus sp.)

Bacillus sp., R. E. Gordon, NRS 1147, NRS 1569

Strains assigned to cluster 29 (Bacillus sp.)

Bacillus sp., R. E. Gordon, NRS 1329, NRS 1572, NRS 1575, NRS 1149 (H. W. Renszer, 1124)

Strains assigned to cluster 30 (Bacillus pantothenticus)

B. pantothenticus, J. R. Norris, BO 183, BO 184

B. pantothenticus, NCIB 8775

B. pantothenticus, WR 3019, WR 3023, WR 3024, WR 3026, WR 3028, WR 3043; soil 
S51-S55

$\mathrm{S} 210$

S213

S498, S499

S485, S486

S264, S265

S266

S416, S418-S422

S417

S56

S114

S341

S197

S226

S378

S493

S496

S25-S29

S35-S37

S38, *S39

$\mathrm{S} 40$

S42, S44-S47

S423-S428

S33, S34

S133, S380

*S433-S436

5441

S48, S49

S134-S136

S301
Table 1 (continued)

Strains assigned to cluster 31 ('Bacillus carotarum' sensu Gibson)

'B. carotarum', J. R. Norris, BO 079, BO 080, BO 081, BO 272, BO 303; T. Gibson, 148, 242, 511, 21 (NCIB 4821), 122

Strains assigned to cluster 32 (Bacillus simplex)

'B. simplex', R. E. Gordon, NRS 335

'B. teres', R. E. Gordon, NRS 986

Strains assigned to cluster 33 (Bacillus sp.)

B. megaterium, R. E. Gordon, NRS 608, NRS 828

Bacillus sp., NRS 1369, NRS 1370

Strains assigned to cluster 34 (Bacillus pulvifaciens)

B. pulvifaciens, WR 3622 (W. C. Haynes, NRS 1283), WR 3623 (W. C. Haynes, NRS 1285)

B. pulvifaciens, H. de Barjac, Institut Pasteur, Paris, strain LES; human origin

Strains assigned to cluster 35 ('Bacillus cascainensis')

'B. cascainensis', ATCC 11968; R. E. Gordon, NRS 1471, NRS 1473, NRS 1474a, NRS 1475, NRS 1474b

'B. cascainensis', R. E. Gordon, NRS 1470

Single-member clusters

'B. carotarum', J. R. Norris, BO 314

'B. epiphytus', J. R. Norris, BO 293

'B. loehnisii', NCTC 4825

'B. macroides', J. R. Norris, BO 204; ATCC 12905

'B. pacificus', J. R. Norris, BO 291

Sporolactobacillus inulinus, NCIB 9743

Bacillus sp., R. E. Gordon, NRS 1562

Bacillus sp., R. E. Gordon, NRS 1573

\section{Cluster-group D}

Strains assigned to cluster 36 ('Bacillus aneurinolyticus')

'B. aneurinolyticus', J. R. Norris, BO 205 (ATCC 12866), BO 206 (NRS 1448), BO 207 (NRS 1450), BO 208 (NRS 1450), BO 209 (NRS 1451)

Strains assigned to cluster 37 (Bacillus brevis)

B. brevis, J. R. Norris, BO 118 (T. Gibson, 539), BO 117 (T. Gibson, 442), BO 270 (NCTC 7096)

B. brevis, NCIB 8803, NCIB 9372

B. brevis, T. R. G. Gray, B54; NCTC 7577

B. brevis, WR 2904, WR 2922, WR 2932, WR 2934, WR 3005; soil

Strains assigned to cluster 38 (Bacillus azotoformans)

B. azotoformans, F. Pichinoty, UER Scientifique de Huming, Marseilles, France, 1, 2, 9, 32, 34, 36; garden soil

Strains assigned to cluster 39 (Bacillus badius/'Bacillus freudenreichii')

B. badius, J. R. Norris, BO 180 (NCTC 10333), BO 201 (M. D. Appleman, NRS 1407)

'B. freudenreichii', J. R. Norris, BO 200 (ATCC 7053), BO 199 (T. Gibson, 68)

Strains assigned to cluster 40 (Bacillus psychrophilus)

B. psychrophilus, J. L. Stokes, Washington State University, USA, W16A (soil), W3 (river water), W5 (soil), W70A

Bacillus sp., J. L. Stokes, T75

Strains assigned to cluster 41 (Bacillus brevis)

B. brevis, WR 3006, WR 3010

Strains assigned to cluster 42 (Bacillus fusiformis)

'B. fusiformis', J. R. Norris, BO 297 (T. Gibson, 1014), WR 2009, WR 2520

B. sphaericus, T. R. G. Gray, B22; NCTC 7582 
Table 1 (continued)

Strains assigned to cluster 43 (Bacillus sphaericus)

$\mathrm{S} 298,{ }^{*} \mathrm{~S} 300, \mathrm{~S} 350$

S302

S303-S307

$\$ 430$

S432

S295

S299

S349

S353

S365

\section{S156}

S164

*S155

S158, S159

S160

S165, S166

*S199, S201

S163

S443, S447, S448

S445, S449

S444

S446

$\mathrm{S} 450$

S454-S456

S459

S451, S452, S457

$\mathrm{S} 458, \mathrm{~S} 460$

S113

S453
B. sphaericus, NCIB 8216, NCIB 9370, G. J. Bonde, 13

B. sphaericus, P. A. Hartman, NRS 348

B. sphaericus, WR 1652, WR 2105, WR 2205, WR 2518, WR 2594

Single-member clusters

B. globisporus, T. L. Stokes, W8

B. insolitus, T. L. Stokes, W16B

'B. repens', J. R. Norris, BO 301

'B. sphaericus var. rotans', NCIB 8867

Bacillus sp., G. J. Bonde, 6 (cluster IIA)

Bacillus sp., G. J. Bonde, 52 (cluster I)

Bacillus sp., G. J. Bonde, 453 (cluster IIAT)

Cluster-group E

Strains assigned to cluster 44 (Bacillus lentus)

B. firmus, R. E. Gordon, NRS 769

B. firmus, WR 3321, R. E. Gordon, NRS 769

B. lentus, NCIB 8773; NRS 670; T. Gibson, 165

B. lentus, R. E. Gordon, NRS 883 (T. Gibson, 165), NRS 1262 (T. Gibson, 258)

B. lentus, J. R. Norris, BO 179; T. Gibson, 238

B. lentus, WR 3322 (R. E. Gordon, NRS 749), WR 3323

Strains assigned to cluster 45 (Bacillus macquariensis)

B. macquariensis, J. R. Norris, BO 188 (NCTC 10419), BO 190 (NCTC 10421)

Single-member cluster

B. lentus, WR 2789

Cluster-group F

Strains assigned to cluster 46 (Bacillus coagulans sensu stricto)

B. coagulans, J. Wolf, University of Leeds, UK, C77, C12, C88

B. coagulans, WR 2972, WR 2822; soil

Strains assigned to cluster 47 (Bacillus coagulans)

B. coagulans, J. Wolf, C32

$B$. coagulans, unknown origin

B. coagulans, WR 2974; mud

Strains assigned to cluster 48 (Bacillus stearothermophilus sensu stricto)

B. stearothermophilus, J. Wolf, T128, T168, T210

B. stearothermophilus, WR 4592

Strains assigned to cluster 49 (Bacillus kaustophilus)

B. stearothermophilus, T1, T39, T349

B. stearothermophilus, WR 4591, WR 4288

Single-member clusters

'B. cirroflagellosus', J. R. Norris, BO 290

B. stearothermophilus, J. Wolf, T76

IIC (B. cereus) were also included in this cluster. B. pumilus strains are readily distinguished from others in the 'subtilis group' by being unable to hydrolyse starch or reduce nitrate.

Several minor clusters contained organisms that shared a high overall similarity with both the $B$. subtilis and $B$. pumilus strains. Three strains of ' $B$. subtilis var. niger' formed a homogeneous cluster in both the $S_{\mathrm{SM}}$ and $D_{\mathrm{P}}$ analyses. These organisms were not pigmented on nutrient agar and showed no consistent single features that allowed them to be distinguished from typical strains of $B$. subtilis. Similarly, a single strain of ' $B$. globigii', often considered to be closely related to either $B$. subtilis or $B$. licheniformis, was recovered as a single-member cluster in this area. Cluster 18 contained two strains received as $B$. subtilis; these organisms were unusual in being 
unable to produce acid from xylose, salicin and mannose. A single marine isolate, a representative of cluster A2 of Boeyé \& Aerts (1976), was recovered between the B. pumilus and $B$. licheniformis clusters. Cluster A2 strains were described as ' $B$. pumilus/B. licheniformis intermediates' in the original publication.

Sixteen strains of $B$. licheniformis, which formed a tight group at $91 \% S_{\mathrm{SM}}$, fused with three additional strains to form cluster 20 . These strains on the periphery of cluster 20 were $B$. subtilis NCIB 9536, originally deposited as 'B. tinakiensis', B. licheniformis NCIB 9668 and a strain received as $B$. subtilis $\mathrm{R} 66-\mathrm{A}$. Cluster 20 conformed to the standard description of $B$. licheniformis.

Eleven strains of $B$. megaterium formed a fairly diffuse taxon (cluster 22) that showed a relatively close affinity with the ' $B$. subtilis group'. This cluster included the type strain of $B$. megaterium, and strains labelled ' $B$. malabarensis' and ' $B$. silvaticus'. The cluster 22 strains formed large cells and conformed to the current description of $B$. megaterium sensu stricto, i.e. they were strictly aerobic, degraded a variety of polysaccharides, were predominantly urease positive and mainly Voges-Proskauer negative. Several minor clusters were associated with the $B$. megaterium taxon. Cluster 21 contained two marine isolates representing Bonde's (1975) group V (B. licheniformis); ' $B$. longissimus' S184 and ' $B$. maroccanus' S202 were recovered as single-member clusters and may represent new centres of variation. Two clusters which fused at $83 \% S_{\mathrm{SM}}$ were peripherally associated with the $B$. megaterium cluster. Cluster 23 contained strains originally labelled ' $B$. agrestis' and ' $B$. flexus'. Strains in these taxa have been considered to belong to the species $B$. megaterium (Gordon et al., 1973). They can be distinguished from $B$. megaterium sensu stricto as they do not hydrolyse aesculin or form acid from arabinose or xylose. A strain of B. firmus and a marine isolate from group B1 of Boeyé \& Aerts (1976), a cluster thought to be related to $B$. firmus, comprised cluster 24 .

Cluster-group C contained B. firmus, B. pantothenticus and a number of unnamed or poorly described strains. Eight strains of $B$. firmus were assigned to a tight taxon (cluster 25) which had the recognized characteristics of this species. These bacteria formed oval, central spores that did not distend the sporangium, were obligately aerobic, produced acid from a restricted range of sugars, and reduced nitrate. 'B. epiphytus' $\mathrm{S} 114$ was recovered on the periphery of the $B$. firmus cluster in the $S_{\mathrm{SM}}$ and $D_{\mathrm{P}}$ analyses but seemed sufficiently dissimilar not to be included. Clusters 26 to 29 contained organisms described by Gordon et al. (1977) as 'B. firmus-B. lentus intermediates'. It is presently difficult to identify features that will distinguish these clusters, although acid production from sugars might be useful. Single-member clusters representing saltmarsh isolates of the so-called ' $B$. firmus- $B$. lentus spectrum' were also recovered in this area of the dendrogram, as was ' $B$. pacificus' S226. Cluster 27 included two marine isolates that were assigned to clusters B2 and B4, both equated with B. firmus, by Boeyé \& Aerts (1976).

Nine strains of $B$. pantothenticus comprised cluster 30 . These $\mathrm{NaCl}$-tolerant bacteria had a variable spore morphology but oval spores predominated. They grew anaerobically and produced acid from a restricted range of sugars. Strains labelled 'Bacillus loehnisii' are generally considered to belong to the species $B$. sphaericus but the single strain bearing this name in the present study was recovered as a single-member cluster near the $B$. pantothenticus taxon. Five strains received as ' $B$. carotarum' constituted cluster 31 , with the sixth strain on the periphery of this cluster. All six strains contained oval central spores with some swelling of the sporangium and produced acid from a limited range of sugars; some were urease positive. ' $B$. simplex' and ' $B$. teres' are often considered to be closely related to $B$. megaterium. Strains bearing these names were assigned to cluster 32 ; they were distinguished from $B$. megaterium by reducing nitrate and failing to hydrolyse aesculin, pullulan or urea. A single isolate of ' $B$. macroides' was recovered adjacent to cluster 33 ; the latter contained two strains received as $B$. megaterium and two ' $B$. firmus-B. lentus intermediates'.

Cluster 34 encompassed three strains of $B$. pulvifaciens which showed $77 \%$ similarity $\left(S_{\mathrm{SM}}\right)$ with the sole isolate of Sporolactobacillus inulinus examined. The B. pulvifaciens strains produced oval, central spores that distended the sporangium, and produced acid from a restricted range of sugars. 


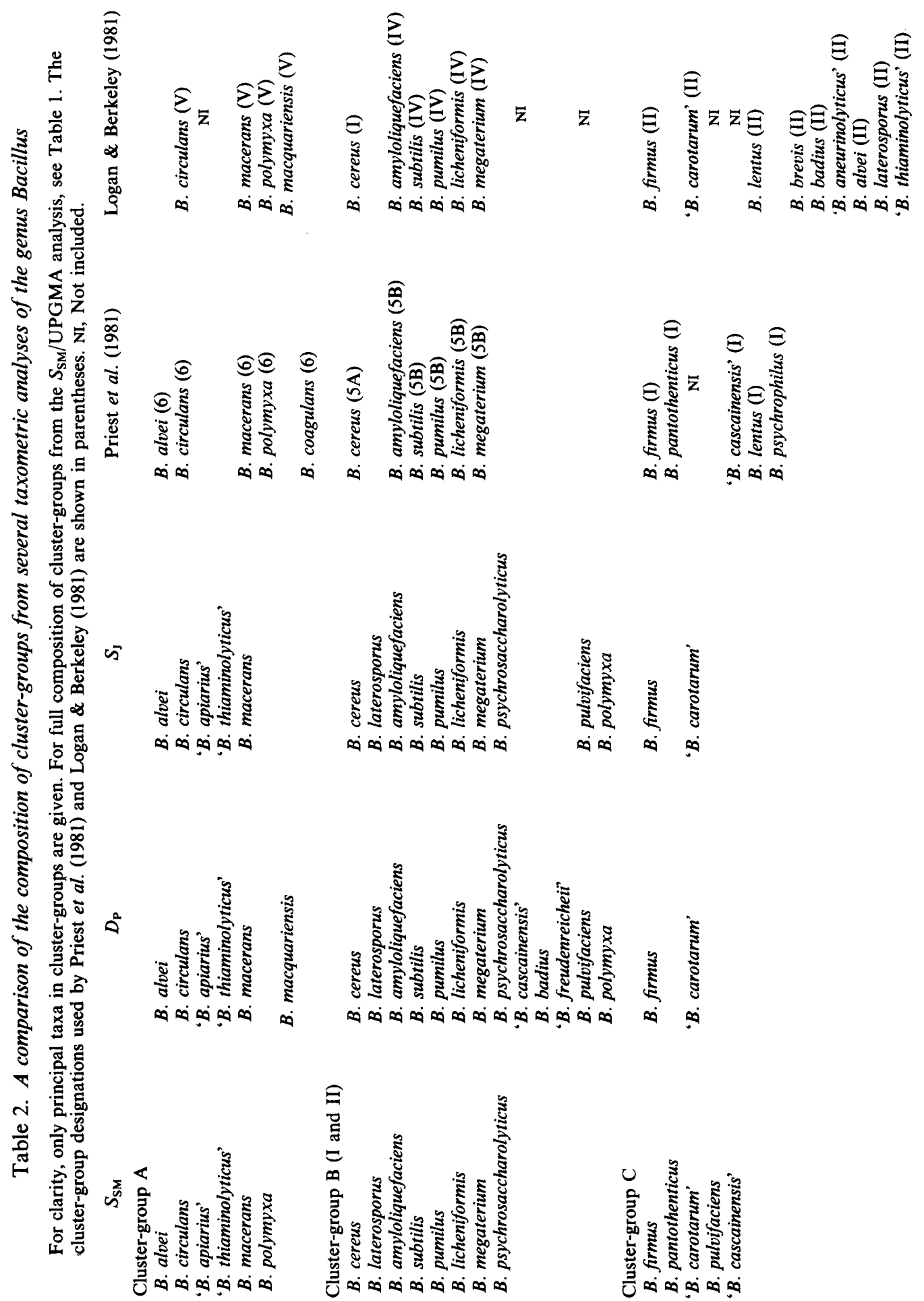



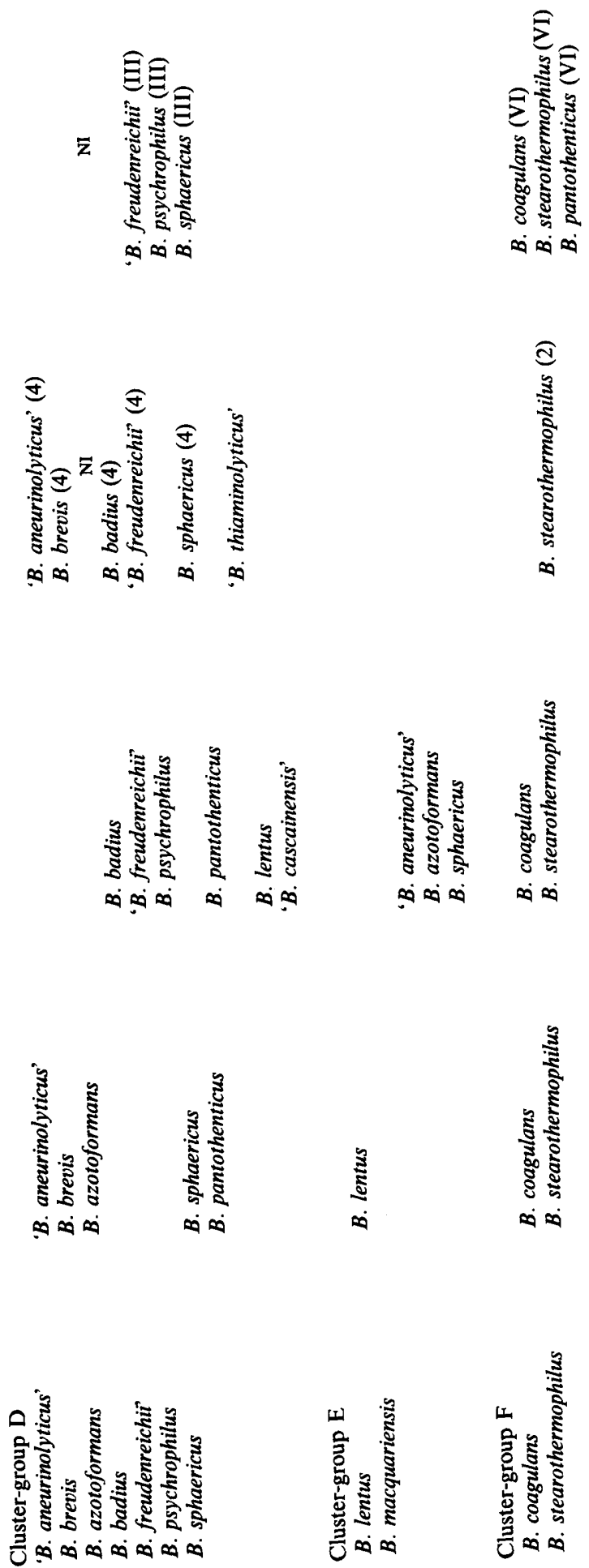
Table 3. Percentage distribution of positive characters to cluster-groups defined at the $70 \%$ level $\left(S_{\mathrm{SM}}\right)$

Cluster-group*... Number of strains. . .

$\begin{array}{lcllll}\text { A } & \text { B } & \text { C } & \text { D } & \text { E } & \text { F } \\ 59 & 154 & 52 & 46 & 10 & 17\end{array}$

Colonial morphology

1. Flat/raised

2. Smooth

3. Rhizoidal

4. Entire

5. Opaque

6. Pigmented

7. Motile colonies

Cellular morphology

8. Length $>3 \mu \mathrm{m}$

9. Diameter $>0.9 \mu \mathrm{m}$

10. Ends round

11. Single

12. Vacuoles present

13. Gram-variable

14. Gram-positive

15. Spores oval

16. Spores round

17. Spores central

18. Spores terminal

19. Spores bulging

20. Sporulation $24 \mathrm{~h}$

21. Sporulation $72 \mathrm{~h}$

22. Sporulation $120 \mathrm{~h}$

23. Sporulation SxA†

Degradation of:
24. Adenine
25. Aesculin
26. Allantoin
27. Arbutin
28. Casein
29. Chitin
30. DNA
31. Elastin
32. Gelatin
33. Guanine
34. Hippurate
35. Lecithin
36. Pectin
37. Pullulan
38. Pustulan
39. RNA
40. Starch
41. Testosterone
42. Tween 20
43. Tween 80
44. Tyrosine
45. Urea

Resistance to $\left(\mu \mathrm{g} \mathrm{ml}^{-1}\right)$ :

46. Benzylpenicillin (8)

47. Benzylpenicillin (4)

48. Chloramphenicol (8)

49. Chloramphenicol (4)

50. Cycloserine (128)

51. Cycloserine (64)

52. Erythromycin (1)

53. Erythromycin $(0.5)$

$\begin{array}{rrrrrr}90 & 74 & 61 & 76 & 60 & 94 \\ 97 & 66 & 100 & 100 & 100 & 94 \\ 0 & 5 & 0 & 0 & 20 & 0 \\ 36 & 32 & 86 & 74 & 80 & 59 \\ 61 & 97 & 96 & 89 & 0 & 35 \\ 3 & 4 & 27 & 0 & 0 & 0 \\ 30 & 0 & 0 & 0 & 0 & 0\end{array}$

$\begin{array}{rrrrrr}75 & 45 & 21 & 67 & 10 & 47 \\ 0 & 42 & 8 & 15 & 0 & 0 \\ 90 & 100 & 98 & 100 & 90 & 100 \\ 76 & 33 & 33 & 76 & 30 & 71 \\ 0 & 41 & 4 & 0 & 0 & 0 \\ 7 & 95 & 73 & 33 & 80 & 59 \\ 0 & 58 & 29 & 6 & 40 & 6 \\ 100 & 99 & 94 & 80 & 100 & 100 \\ 0 & 0 & 11 & 28 & 0 & 0 \\ 24 & 99 & 65 & 59 & 100 & 35 \\ 75 & 3 & 21 & 50 & 0 & 59 \\ 47 & 92 & 25 & 87 & 20 & 76 \\ 10 & 17 & 0 & 35 & 0 & 100 \\ 86 & 88 & 70 & 87 & 60 & 100 \\ 100 & 99 & 81 & 96 & 80 & 100 \\ 100 & 99 & 94 & 98 & 100 & 100\end{array}$

$\begin{array}{rrrrrr}0 & 15 & 2 & 28 & 0 & 18 \\ 100 & 99 & 65 & 7 & 100 & 94 \\ 7 & 16 & 10 & 15 & 0 & 0 \\ 100 & 100 & 38 & 15 & 100 & 100 \\ 68 & 100 & 100 & 61 & 0 & 41 \\ 28 & 38 & 0 & 0 & 0 & 0 \\ 68 & 95 & 94 & 100 & 0 & 100 \\ 10 & 53 & 13 & 17 & 0 & 0 \\ 90 & 100 & 100 & 72 & 0 & 71 \\ 0 & 1 & 13 & 0 & 0 & 0 \\ 57 & 21 & 52 & 39 & 80 & 100 \\ 52 & 88 & 71 & 28 & 0 & 0 \\ 15 & 28 & 4 & 0 & 0 & 0 \\ 83 & 64 & 60 & 0 & 100 & 53 \\ 12 & 1 & 0 & 0 & 0 & 0 \\ 80 & 100 & 81 & 67 & 0 & 88 \\ 98 & 75 & 46 & 0 & 100 & 100 \\ 85 & 1 & 2 & 50 & 30 & 29 \\ 100 & 100 & 98 & 72 & 80 & 100 \\ 73 & 80 & 86 & 65 & 80 & 47 \\ 12 & 32 & 27 & 28 & 0 & 0 \\ 20 & 21 & 4 & 26 & 80 & 0\end{array}$

$\begin{array}{rrrrrr}3 & 42 & 11 & 0 & 20 & 0 \\ 20 & 52 & 11 & 6 & 30 & 0 \\ 15 & 25 & 21 & 13 & 0 & 0 \\ 19 & 35 & 29 & 24 & 0 & 0 \\ 73 & 35 & 13 & 65 & 10 & 0 \\ 86 & 56 & 50 & 85 & 30 & 0 \\ 37 & 30 & 35 & 37 & 80 & 6 \\ 66 & 34 & 23 & 30 & 80 & 6\end{array}$


Table 3 (continued)

Cluste
Number
in (64)
an (32)
acid (32)
acid (16)
in (16)
in (8)
$(0 \cdot 25)$
in $(0 \cdot 125)$
$y c i n(16)$
$y c i n(8)$
ine (2)
ine (1)

\begin{tabular}{lrrrrr} 
A & B & C & D & \multicolumn{1}{c}{ E } & F \\
59 & 154 & 52 & 46 & 10 & 17 \\
73 & 61 & 27 & 78 & 10 & 0 \\
83 & 73 & 35 & 83 & 30 & 0 \\
22 & 7 & 54 & 72 & 90 & 100 \\
17 & 22 & 65 & 83 & 100 & 100 \\
80 & 89 & 6 & 50 & 0 & 6 \\
88 & 90 & 27 & 72 & 10 & 6 \\
22 & 14 & 25 & 4 & 0 & 0 \\
34 & 43 & 31 & 11 & 0 & 0 \\
49 & 35 & 0 & 59 & 100 & 0 \\
66 & 52 & 4 & 61 & 100 & 0 \\
46 & 43 & 0 & 20 & 0 & 0 \\
49 & 72 & 0 & 37 & 0 & 0
\end{tabular}

\section{Acid from: \\ 66. Adonitol \\ 67. Arabinose \\ 68. Cellobiose \\ 69. Dulcitol \\ 70. Erythritol \\ 71. Fructose \\ 72. Galactose \\ 73. Glucose \\ 74. Glycerol \\ 75. meso-Inositol \\ 76. Lactose \\ 77. Maltose \\ 78. Mannitol \\ 79. Mannose \\ 80. Raffinose \\ 81. Rhamnose \\ 82. Salicin \\ 83. Sorbitol \\ 84. Sucrose \\ 85. Trehalose \\ 86. Xylose}

$\begin{array}{rrrrrr}36 & 0 & 0 & 0 & 0 & 6 \\ 49 & 35 & 0 & 0 & 0 & 0 \\ 97 & 96 & 40 & 2 & 60 & 35 \\ 0 & 0 & 2 & 0 & 0 & 0 \\ 2 & 0 & 11 & 0 & 0 & 18 \\ 75 & 94 & 40 & 24 & 30 & 71 \\ 85 & 40 & 25 & 0 & 60 & 23 \\ 98 & 99 & 79 & 15 & 80 & 100 \\ 90 & 94 & 71 & 59 & 20 & 41 \\ 30 & 35 & 2 & 0 & 0 & 29 \\ 73 & 23 & 19 & 0 & 80 & 0 \\ 100 & 93 & 60 & 9 & 50 & 88 \\ 60 & 42 & 63 & 2 & 70 & 47 \\ 63 & 59 & 17 & 0 & 70 & 71 \\ 97 & 48 & 21 & 2 & 60 & 23 \\ 7 & 4 & 11 & 0 & 10 & 0 \\ 76 & 94 & 19 & 0 & 60 & 18 \\ 25 & 26 & 13 & 0 & 10 & 0 \\ 100 & 80 & 75 & 2 & 40 & 71 \\ 93 & 98 & 75 & 2 & 10 & 88 \\ 63 & 43 & 0 & 0 & 50 & 35\end{array}$

Utilization of:

87. Acetate

88. Citrate

89. Formate

90. Gluconate

91. Lactate

92. Malonate

93. Succinate

$\begin{array}{rrrrrr}19 & 55 & 48 & 63 & 40 & 12 \\ 12 & 74 & 44 & 30 & 10 & 59 \\ 34 & 77 & 44 & 50 & 20 & 18 \\ 49 & 47 & 27 & 4 & 30 & 6 \\ 24 & 62 & 31 & 37 & 20 & 35 \\ 5 & 10 & 21 & 4 & 0 & 6 \\ 32 & 41 & 60 & 50 & 10 & 29\end{array}$

\section{Growth at :}

94. $\mathrm{pH} 4.5$

95. $\mathrm{pH} 6.0$

96. $\mathrm{pH} 7 \cdot 2$

97. $\mathrm{pH} 8.0$

98. $\mathrm{pH} 9.5$

99. $5^{\circ} \mathrm{C}$

100. $17^{\circ} \mathrm{C}$

101. $37^{\circ} \mathrm{C}$

102. $50^{\circ} \mathrm{C}$

103. $65^{\circ} \mathrm{C}$

$\begin{array}{rrrrrr}0 & 15 & 23 & 0 & 10 & 12 \\ 78 & 96 & 98 & 87 & 100 & 94 \\ 100 & 100 & 100 & 100 & 100 & 82 \\ 100 & 100 & 100 & 100 & 100 & 35 \\ 95 & 99 & 100 & 80 & 100 & 0 \\ 12 & 3 & 6 & 9 & 20 & 0 \\ 78 & 97 & 94 & 72 & 100 & 6 \\ 100 & 100 & 100 & 89 & 90 & 94 \\ 27 & 34 & 25 & 43 & 0 & 100 \\ 0 & 0 & 0 & 0 & 0 & 53\end{array}$

Growth in $(\%, w / v)$ :

$\begin{array}{lrrrrrr}\text { 104. } \mathrm{NaCl}(2) & 83 & 100 & 100 & 87 & 100 & 47 \\ \text { 105. } \mathrm{NaCl}(5) & 58 & 99 & 96 & 70 & 80 & 29 \\ \text { 106. } \mathrm{NaCl}(10) & 2 & 52 & 79 & 0 & 20 & 6\end{array}$


Table 3 (continued)

$\begin{array}{ccccccc}\text { Cluster-group*... } & \text { A } & \text { B } & \text { C } & \text { D } & \text { E } & \text { F } \\ \text { Number of strains. . } & 59 & 154 & 52 & 46 & 10 & 17\end{array}$

Miscellaneous tests:
107. Anaerobic growth
108. Gas from glucose
109. Dihydroxyacetone production
110. Indole production
111. Growth on MacConkey agar
112. Methyl red test
113. Nitrate reduction
114. ONPG
115. Oxidase
116. Phenylalanine deamination
117. Phosphatase
118. Voges-Proskauer reaction

$\begin{array}{rrrrrr}98 & 52 & 23 & 0 & 20 & 57 \\ 36 & 0 & 0 & 0 & 0 & 0 \\ 41 & 40 & 0 & 4 & 0 & 0 \\ 27 & 2 & 0 & 2 & 0 & 0 \\ 41 & 68 & 77 & 63 & 80 & 35 \\ 78 & 76 & 6 & 0 & 20 & 47 \\ 75 & 75 & 52 & 67 & 30 & 100 \\ 100 & 57 & 48 & 13 & 100 & 47 \\ 72 & 29 & 6 & 41 & 80 & 0 \\ 0 & 29 & 32 & 6 & 0 & 0 \\ 37 & 71 & 13 & 20 & 20 & 65 \\ 36 & 80 & 0 & 0 & 0 & 18\end{array}$

* For ease of computation, these cluster-groups do not take into account data for single-member clusters. + SxA, soil extract agar.

$\ddagger$ ONPG, $\alpha$-nitrophenyl $\beta$-D-galactoside.

The final phenon in cluster-group C comprised seven strains originally described as 'Krusella cascainensis' (Castellani, 1954) but subsequently transferred to Bacillus as ' $B$. cascainensis' (Castellani, 1955). Most of these bacteria formed endospores that were oval and central but did not swell the sporangium; their other characteristics are given in Table 4.

Cluster-group D contained the alkali-forming strains that have limited, if any, reaction in sugar-containing media. Five strains of ' $B$. aneurinolyticus' formed a homogeneous group (cluster 36 ) that was closely related to $B$. brevis (cluster 37 ). Both of these taxa accommodated strains with oval central spores that distended the sporangium. They were obligate aerobes, reduced nitrate and with the occasional exception did not produce acid from carbohydrates. The two species were distinguished by the failure of ' $B$. aneurinolyticus' strains to grow in $5 \%(\mathrm{w} / \mathrm{v}) \mathrm{NaCl}$ or to hydrolyse casein, gelatin or hippurate. Six strains of B. azotoformans were recovered close to $B$. brevis in cluster 38 . These species had many features in common, but $B$. azotoformans strains can be distinguished as they are unable to grow at $50^{\circ} \mathrm{C}$ and fail to hydrolyse casein, gelatin, hippurate or RNA. In the $S_{\mathrm{SM}} / \mathrm{UPGMA}$ analysis, cluster 39 contained strains of $B$. badius and ' $B$. freudenreichii' but these taxa were separated in the analyses based on $S_{\mathrm{J}}$ and $D_{\mathrm{P}}$ coefficients. The $B$. badius strains were negative for nitrate reduction and urease production, but hydrolysed casein and gelatin; the ' $B$. freudenreichii' strains gave the opposite reactions.

Five psychrophilic isolates were assigned to cluster $40, B$. psychrophilus. These bacteria produced spherical spores that distended the sporangium and most grew at $5{ }^{\circ} \mathrm{C}$ but not at $37^{\circ} \mathrm{C}$. Cluster 41 , which was recovered in all three analyses, contained two $B$. brevis strains. Morphologically similar to $B$. brevis sensu stricto, these strains differed by degrading adenine, allantoin and elastin, and were also urease positive and did not reduce nitrate.

The $B$. sphaericus and ' $B$. sphaericus var. fusiformis' strains were recovered in two discrete clusters in all three analyses, suggesting that the latter should be given species status as $B$. fusiformis. The remaining strains in cluster-group $\mathrm{D}$ were recovered as single-member clusters and included 'B. sphaericus var. rotans' S299, B. globisporus S430, B. insolitus S432, 'B. repens' S295 and three marine isolates.

Cluster-group E contained a single major cluster, B. lentus. The bacteria in this taxon had limited action on macromolecules, formed acid from few sugars other than glucose and produced oval central spores that did not swell the sporangium. Two strains of $B$. macquariensis were recovered in a minor cluster adjacent to $B$. lentus.

The thermophilic bacilli were recovered in cluster-group F. Eight strains of $B$. coagulans were recovered in two clusters, one of which, cluster 46 , conformed to $B$. coagulans sensu stricto (Wolf 
Type B; Wolf \& Sharp, 1981). Similarly, the B. stearothermophilus strains were assigned to two major phena. Cluster 48 contained strains belonging to Groups 2 and 3 (B. stearothermophilus Donk) of Walker \& Wolf (1971). These two groups of bacteria fused at $84 \% S_{\mathrm{SM}}$ but were assigned to separate clusters in the $S_{\mathrm{J}}$ analysis. Thus, the characteristics shown in Table 4 may not be typical for $B$. stearothermophilus sensu stricto. Cluster 49 equated with Group 1 of Walker \& Wolf (1971) ('B. kaustophilus').

\section{DISCUSSION}

It is encouraging that the three analyses presented here and the two previous comprehensive taxometric studies of bacilli (Logan \& Berkeley, 1981; Priest et al., 1981) are essentially congruous despite the use of widely different data bases. Indeed, the assignment of species to cluster-groups seems to reflect a natural classification that is largely consistent with DNA base composition (Priest, 1981). The cluster-groups can be equated with genera in some groups of bacteria, but additional data derived from 16S rRNA sequencing or hybridization studies are needed before any dismemberment of the genus Bacillus can be proposed with confidence. For the present, the cluster-groups should be used as a framework for further taxonomic studies, and to this end their characteristics have been considered above. The ensuing discussion concentrates on species of Bacillus that currently present taxonomic problems.

Cluster-group $A$. This study confirms the heterogeneity of strains currently classified as $B$. circulans. Gibson \& Topping (1938) described B. circulans as a 'complex' rather than a species, a view that persisted for some time (Proom \& Knight, 1955; Wolf \& Chowdbury, 1971; Gibson \& Gordon, 1974). It is now apparent that the description of $B$. circulans encompasses a variety of genotypically unrelated bacteria. The $\mathrm{mol} \% \mathrm{G}+\mathrm{C}$ of 123 strains identified as $B$. circulans varied between 37 and 61 (Nakamura \& Swezey, 1983a), and in DNA reassociation experiments nearly half of these bacteria were assigned to 10 homology groups, while the remaining 61 strains were unclassified (Nakamura \& Swezey, 1983b). From these studies, four species names previously considered as synonyms of $\boldsymbol{B}$. circulans, namely $\boldsymbol{B}$. amylolyticus, $\boldsymbol{B}$. lautus, B. pabuli and B. validus, were reintroduced (Nakamura, 1984a). Our numerical classification included few of the strains examined by Nakamura \& Swezey $(1983 a, b)$ but it is possible to equate the two studies. Cluster 6 contained the type strain and has properties in accord with those of $B$. circulans sensu stricto (Nakamura, 1984a). Further evidence for homogeneity of this cluster is indicated by the inclusion of strain S109 (NCIB 9555), which originally bore the name ' $B$. aporrhoeus' but is now considered to be a synonym of $B$. circulans (Gordon et al., 1973) and shares 50 to $60 \%$ DNA sequence homology with the type strain of $B$. circulans (Nakamura \& Swezey, $1983 \mathrm{~b}$ ). Cluster 7 is similar to $B$. pabuli in most respects. Similarly, strains assigned to cluster 9 have much in common with $B$. amylolyticus and cluster 10 can perhaps be equated with $B$. lautus, although it contains strains that hydrolyse Tween 80 and do not produce acid from rhamnose. The numerical classification also underpins the taxonomic integrity of $B$. alvei, B. macerans and $B$. polymyxa. It is also evident that strains of ' $B$. apiarius' and ' $B$. thiaminolyticus' form well-circumscribed taxa which may merit species status when DNA base composition data become available.

Cluster-group B. DNA reassociation studies support the view that $B$. cereus, B. mycoides and B. thuringiensis comprise a single species (Somerville \& Jones, 1972; Seki et al., 1978). In this respect, it is interesting that crystal toxin synthesis is often plasmid-encoded and transmissible from $B$. thuringiensis to $B$. cereus by 'conjugation' (Gonzalez et al., 1982). The numerical phenetic data underline the close relationship between $B$. cereus and $B$. thuringiensis, although strains bearing these names were largely allocated to separate subclusters within cluster 11 . Some strains of $B$. cereus are responsible for diarrhoeal and emetic types of food poisoning (Gilbert, 1979) and others for quite severe medical and veterinary pathogenic conditions (Turnbull et al., 1979); a serotyping scheme has been developed for the identification of these strains (Kramer $e t$ $a l ., 1982$ ). It has been claimed that strains of $B$. cereus responsible for the emetic form of foodpoisoning can be distinguished from diarrhoeal and non-food-poisoning strains by numerical 
Table 4. Percentage distribution of positive characters to major clusters defined at the $83 \%$ level $\left(S_{\mathrm{SM}}\right)$

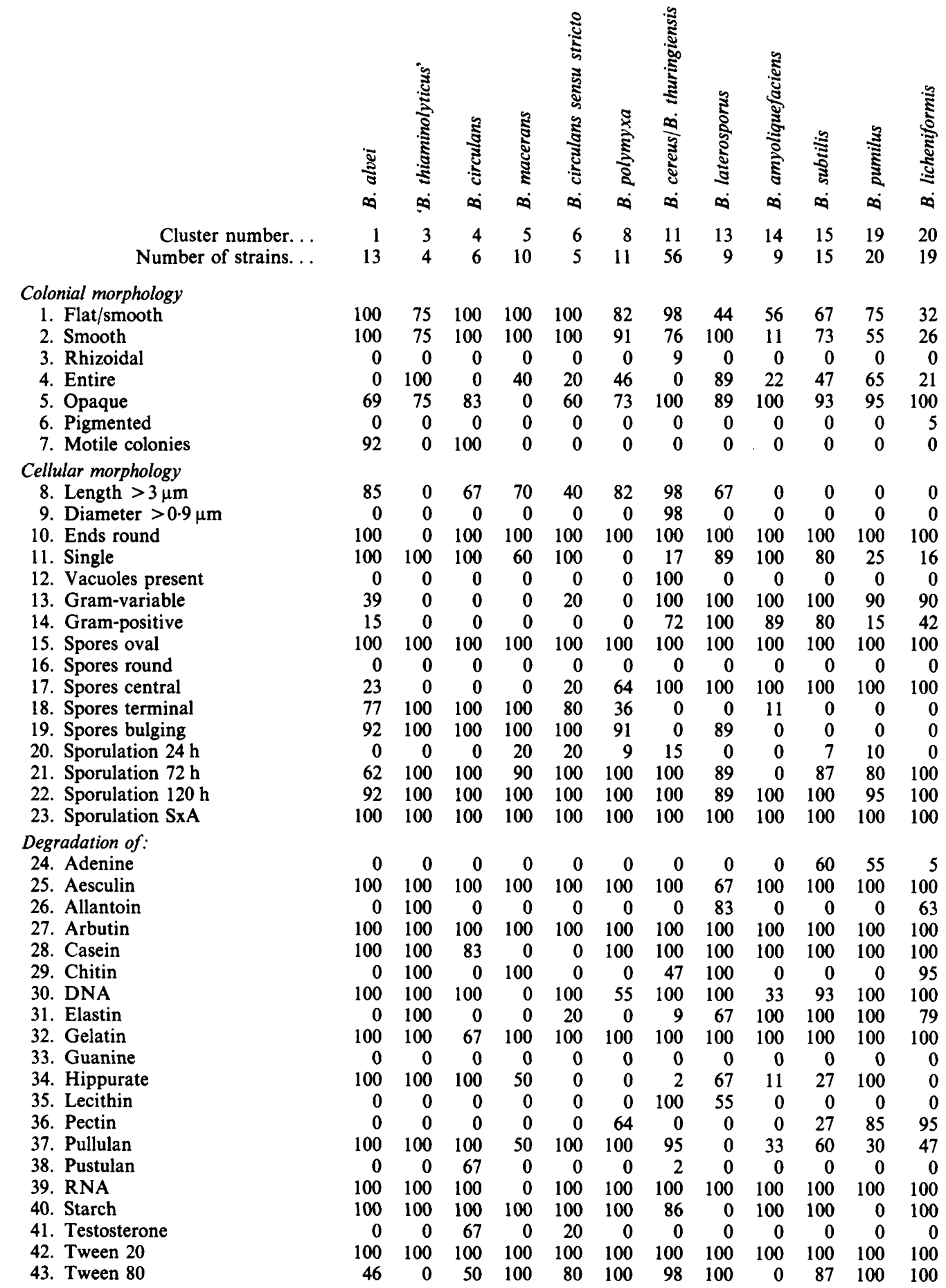


Table 4 (continued)

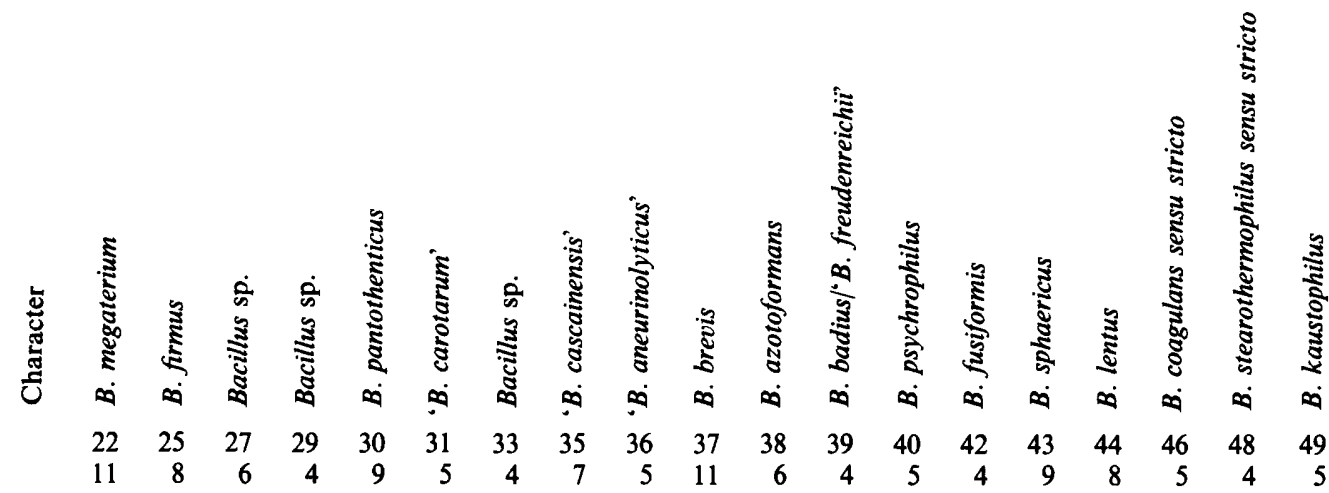

$\begin{array}{llllllllllllllllllll}\text { 1. } & 73 & 88 & 17 & 75 & 100 & 0 & 0 & 100 & 100 & 91 & 83 & 50 & 100 & 25 & 56 & 63 & 100 & 75 & 100\end{array}$

2. $\quad \begin{array}{rrrrrrrrrrrrrrrrrrrr}100 & 100 & 100 & 100 & 100 & 100 & 100 & 100 & 100 & 100 & 100 & 100 & 100 & 100 & 100 & 100 & 100 & 100 & 80\end{array}$

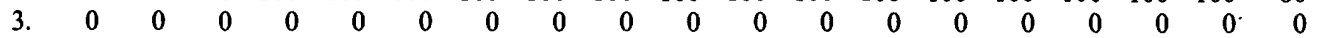

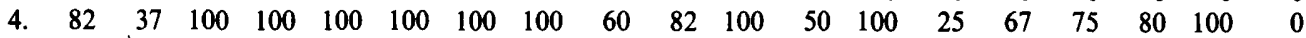

5. $100 \begin{array}{lllllllllllllllllll}100 & 100 & 100 & 100 & 100 & 100 & 43 & 100 & 100 & 83 & 100 & 60 & 100 & 100 & 0 & 80 & 25 & 0\end{array}$

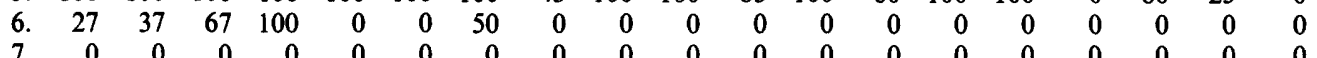

$\begin{array}{lllllllllllllllllllll}8 . & 54 & 0 & 0 & 25 & 0 & 80 & 75 & 0 & 100 & 27 & 100 & 100 & 0 & 100 & 89 & 0 & 100 & 0 & 0\end{array}$

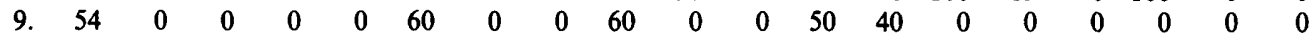

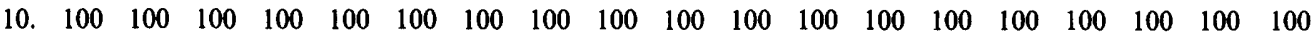

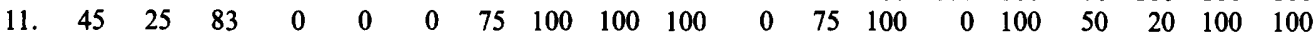

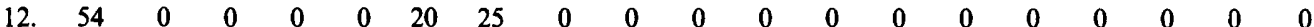

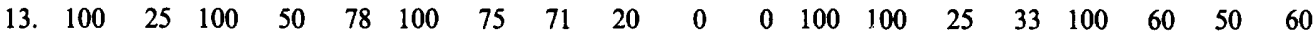

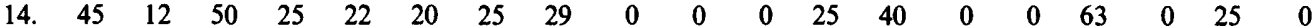

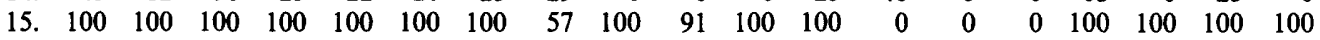

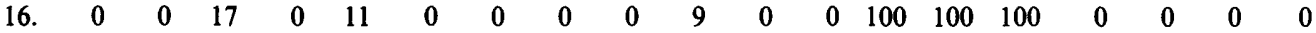

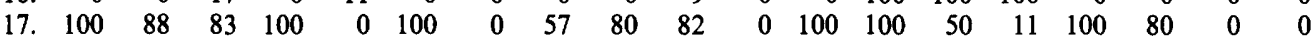

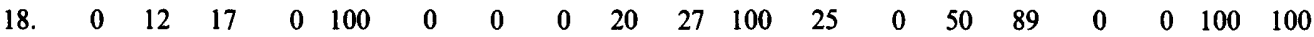

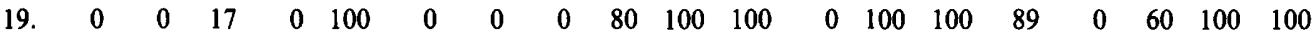

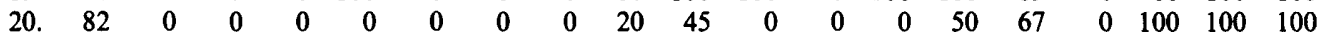

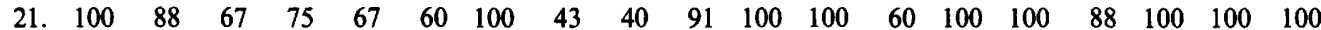

$\begin{array}{llllllllllllllllllll}22 . & 100 & 88 & 67 & 75 & 89 & 60 & 100 & 57 & 60 & 100 & 100 & 100 & 100 & 100 & 100 & 100 & 100 & 100 & 100\end{array}$

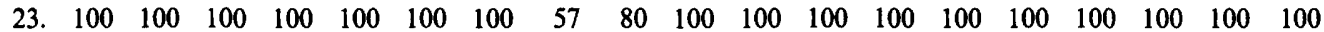

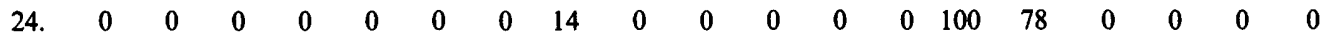

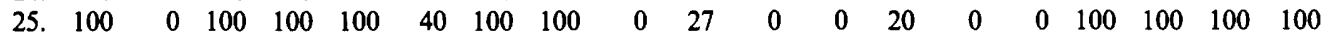

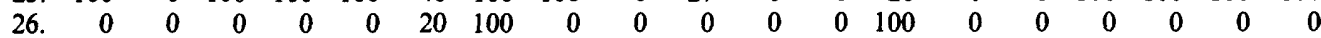

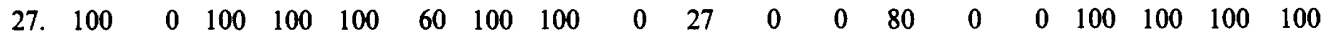
28. $100 \begin{array}{lllllllllllllllllll}100 & 100 & 100 & 100 & 100 & 100 & 100 & 0 & 100 & 0 & 50 & 100 & 100 & 89 & 0 & 0 & 75 & 20\end{array}$

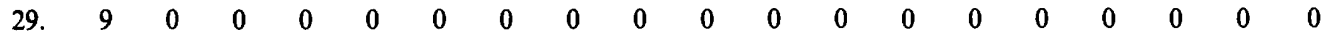

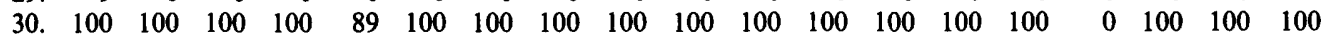
31. $\quad 0 \begin{array}{rrrrrrrrrrrrrrrrrr}50 & 33 & 25 & 0 & 0 & 0 & 0 & 0 & 0 & 0 & 0 & 0 & 75 & 33 & 0 & 0 & 0 & 0\end{array}$ 32. $100 \begin{array}{rlrrrrrrrrrrrrrrrrrr}100 & 100 & 100 & 100 & 100 & 100 & 100 & 0 & 100 & 0 & 50 & 100 & 100 & 100 & 0 & 0 & 100 & 100\end{array}$

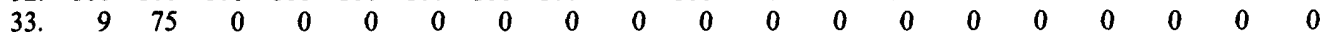
$\begin{array}{llllllllllllllllllll}34 & 9 & 88 & 0 & 50 & 0 & 100 & 100 & 86 & 0 & 91 & 0 & 50 & 100 & 0 & 11 & 100 & 100 & 100 & 100\end{array}$

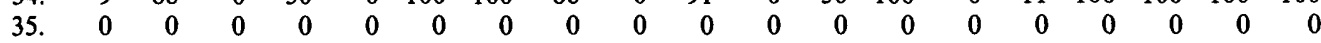

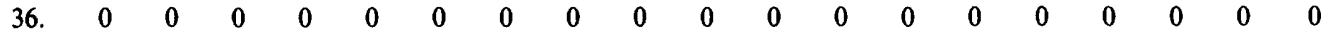
$\begin{array}{llllllllllllllllllll}37 . & 91 & 100 & 83 & 100 & 78 & 0 & 25 & 14 & 0 & 0 & 0 & 0 & 0 & 0 & 0 & 100 & 0 & 100 & 100\end{array}$

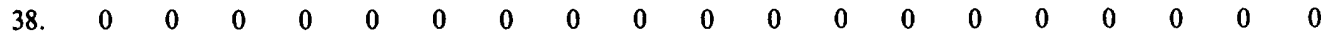

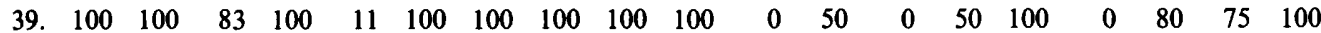

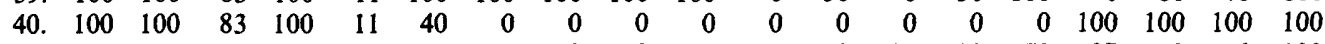
41. $\quad 0 \begin{array}{llllllllllllllllll}0 & 0 & 0 & 11 & 0 & 0 & 0 & 0 & 55 & 0 & 0 & 80 & 100 & 78 & 37 & 0 & 0 & 100\end{array}$

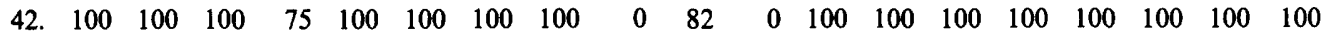

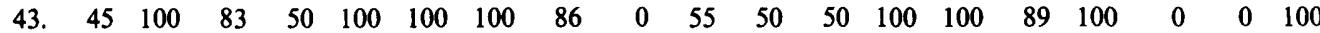




\section{Table 4 (continued)}

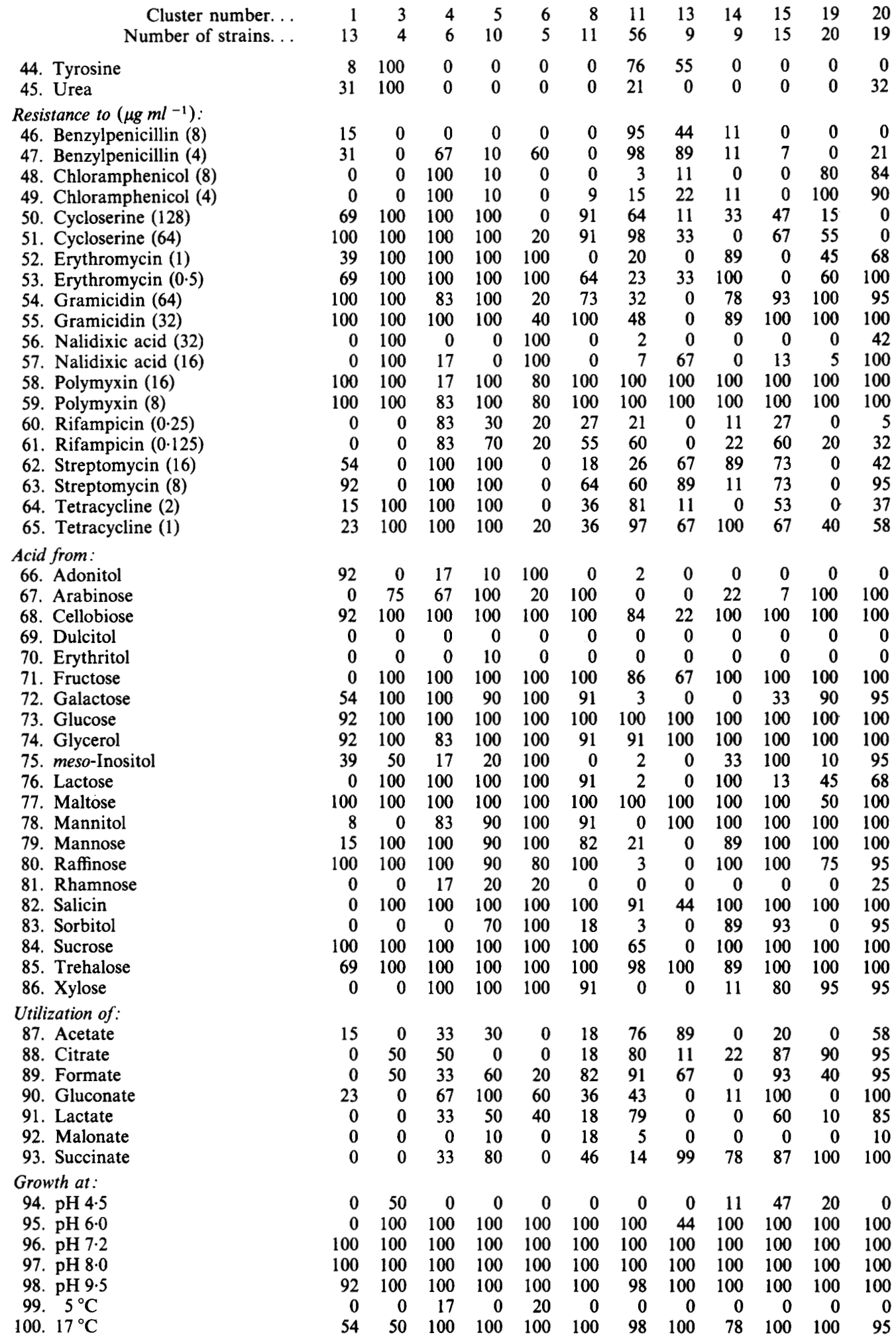


$\begin{array}{rrrrrrrrrrrrrrrrrrr}22 & 25 & 27 & 29 & 30 & 31 & 33 & 35 & 36 & 37 & 38 & 39 & 40 & 42 & 43 & 44 & 46 & 48 & 49 \\ 11 & 8 & 6 & 4 & 9 & 5 & 4 & 7 & 5 & 11 & 6 & 4 & 5 & 4 & 9 & 8 & 5 & 4 & 5\end{array}$

$\begin{array}{rrrrrrrrrrrrrrrrrrrr}44 . & 0 & 0 & 0 & 0 & 56 & 100 & 50 & 0 & 100 & 36 & 0 & 100 & 0 & 0 & 0 & 0 & 0 & 0 & 0 \\ 45 . & 82 & 0 & 0 & 0 & 0 & 60 & 0 & 0 & 0 & 0 & 0 & 50 & 100 & 75 & 0 & 100 & 0 & 0 & 0\end{array}$

$\begin{array}{rrrrrrrrrrrrrrrrrrrr}46 . & 9 & 0 & 0 & 0 & 0 & 0 & 100 & 0 & 0 & 0 & 0 & 0 & 0 & 0 & 0 & 25 & 0 & 0 & 0 \\ 47 & 45 & 0 & 0 & 0 & 0 & 0 & 100 & 0 & 0 & 0 & 0 & 0 & 0 & 25 & 22 & 37 & 0 & 0 & 0\end{array}$

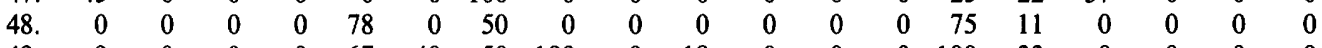

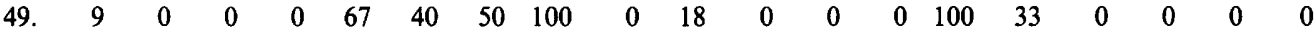

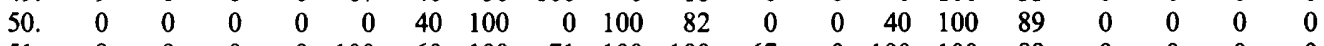
$\begin{array}{llllllllllllllllllll}51 . & 9 & 0 & 0 & 0 & 100 & 60 & 100 & 71 & 100 & 100 & 67 & 0 & 100 & 100 & 89 & 0 & 0 & 0 & 0\end{array}$ $\begin{array}{llllllllllllllllllll}52 . & 9 & 100 & 0 & 0 & 67 & 0 & 50 & 0 & 0 & 18 & 100 & 0 & 0 & 100 & 33 & 100 & 20 & 0 & 0\end{array}$ $\begin{array}{rrrrrrrrrrrrrrrrrrrr}53 . & 27 & 100 & 0 & 0 & 0 & 0 & 50 & 0 & 0 & 18 & 100 & 0 & 0 & 25 & 33 & 100 & 20 & 0 & 0\end{array}$ $\begin{array}{rrrrrrrrrrrrrrrrrrrrr}54 & 27 & 0 & 0 & 25 & 0 & 20 & 50 & 100 & 100 & 100 & 100 & 25 & 60 & 25 & 78 & 0 & 0 & 0 & 0\end{array}$ $\begin{array}{rrrrrrrrrrrrrrrrrrrr}55 . & 73 & 0 & 17 & 50 & 11 & 60 & 50 & 100 & 100 & 100 & 100 & 25 & 80 & 25 & 89 & 25 & 0 & 0 & 0\end{array}$ $\begin{array}{llllllllllllllllllll}56 . & 0 & 12 & 0 & 0 & 100 & 100 & 100 & 100 & 100 & 73 & 100 & 0 & 0 & 100 & 100 & 100 & 100 & 100 & 100\end{array}$ $\begin{array}{llllllllllllllllllll}57 . & 0 & 50 & 0 & 0 & 100 & 100 & 100 & 100 & 100 & 100 & 100 & 0 & 20 & 100 & 100 & 100 & 100 & 100 & 100\end{array}$ $\begin{array}{lllllllllllllllllllll}58 . & 0 & 0 & 0 & 0 & 0 & 0 & 0 & 0 & 100 & 100 & 0 & 0 & 0 & 25 & 44 & 0 & 20 & 0 & 0\end{array}$ $\begin{array}{rrrrrrrrrrrrrrrrrrrr}59 . & 9 & 12 & 0 & 0 & 33 & 0 & 0 & 100 & 100 & 100 & 33 & 0 & 0 & 100 & 100 & 0 & 20 & 0 & 0\end{array}$ $\begin{array}{llllllllllllllllllll}60 . & 0 & 75 & 0 & 25 & 0 & 20 & 50 & 0 & 0 & 0 & 0 & 0 & 0 & 0 & 11 & 0 & 0 & 0 & 0\end{array}$ $\begin{array}{llllllllllllllllllll}61 . & 0 & 88 & 0 & 25 & 0 & 100 & 0 & 0 & 0 & 0 & 0 & 0 & 0 & 25 & 33 & 0 & 0 & 0 & 0\end{array}$

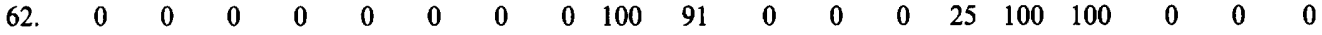

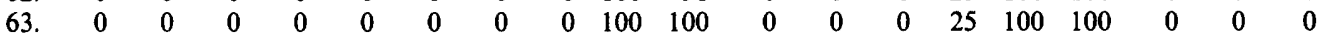
$\begin{array}{rrrrrrrrrrrrrrrrrrrr}64 . & 0 & 0 & 0 & 0 & 0 & 0 & 0 & 0 & 0 & 27 & 0 & 0 & 0 & 0 & 67 & 0 & 0 & 0 & 0 \\ 65 & 9 & 0 & 0 & 0 & 0 & 0 & 0 & 14 & 0 & 64 & 0 & 0 & 20 & 0 & 100 & 0 & 0 & 0 & 0\end{array}$

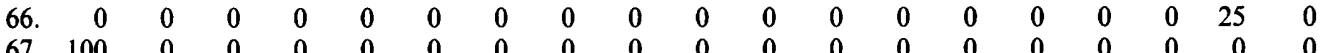

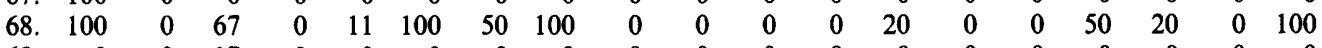

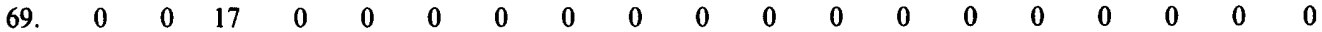
$\begin{array}{llllllllllllllllllll}70 . & 0 & 0 & 0 & 0 & 0 & 0 & 0 & 0 & 0 & 0 & 0 & 0 & 0 & 0 & 0 & 0 & 0 & 0 & 0\end{array}$ $\begin{array}{rrrrrrrrrrrrrrrrrrrr}\text { 71. } & 100 & 12 & 67 & 0 & 67 & 80 & 0 & 100 & 0 & 73 & 0 & 0 & 60 & 0 & 0 & 0 & 60 & 100 & 100\end{array}$

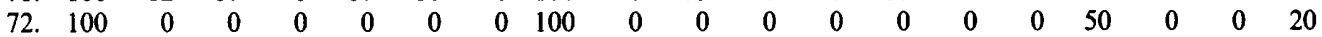
$\begin{array}{llllllllllllllllllll}\text { 73. } & 100 & 100 & 100 & 0 & 78 & 100 & 0 & 100 & 40 & 9 & 17 & 0 & 60 & 0 & 0 & 100 & 100 & 100 & 100\end{array}$ $\begin{array}{llllllllllllllllllll}74 . & 100 & 88 & 83 & 50 & 11 & 100 & 75 & 100 & 100 & 82 & 50 & 50 & 60 & 0 & 33 & 12 & 0 & 25 & 80\end{array}$

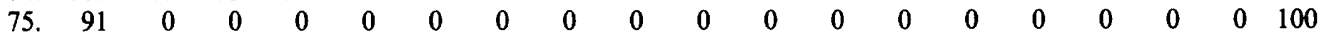

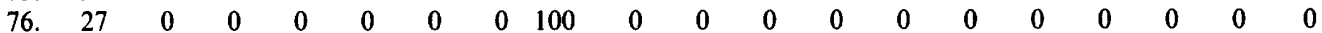
$\begin{array}{llllllllllllllllllll}\text { 77. } & 100 & 100 & 100 & 50 & 22 & 80 & 50 & 0 & 20 & 0 & 0 & 0 & 60 & 0 & 0 & 37 & 60 & 100 & 100\end{array}$ $\begin{array}{rrrrrrrrrrrrrrrrrrrrr}78 & 100 & 88 & 100 & 50 & 0 & 100 & 75 & 100 & 0 & 0 & 0 & 0 & 0 & 25 & 0 & 88 & 0 & 75 & 100\end{array}$

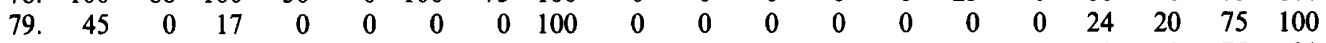
$\begin{array}{rlllllllllllllllllll}\text { 80. } & 0 & 0 & 0 & 0 & 0 & 60 & 0 & 100 & 0 & 9 & 0 & 0 & 0 & 0 & 0 & 0 & 0 & 75 & 20\end{array}$ $\begin{array}{rrrrrrrrrrrrrrrrrrrr}81 . & 0 & 0 & 0 & 0 & 0 & 0 & 0 & 86 & 0 & 0 & 0 & 0 & 0 & 0 & 0 & 0 & 0 & 0 & 0\end{array}$ 82. $100 \begin{array}{lllllllllllllllllll}12 & 0 & 0 & 11 & 0 & 0 & 100 & 0 & 0 & 0 & 0 & 0 & 0 & 0 & 50 & 0 & 25 & 40\end{array}$

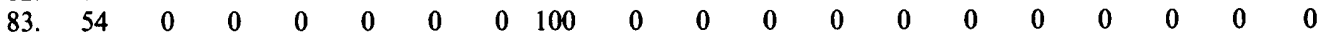

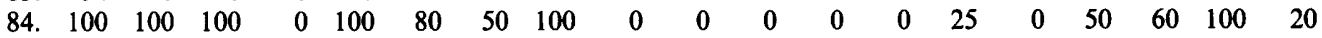
$\begin{array}{llllllllllllllllllll}85 & 100 & 12 & 67 & 75 & 100 & 100 & 50 & 100 & 0 & 0 & 0 & 0 & 20 & 0 & 0 & 12 & 60 & 100 & 100\end{array}$

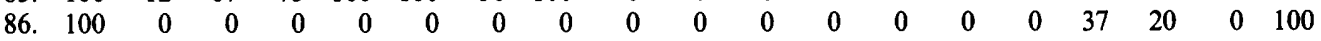

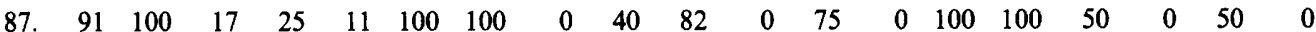

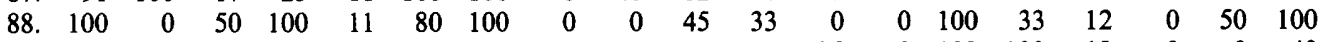
89. $100 \begin{array}{lllllllllllllllllll} & 100 & 0 & 0 & 11 & 100 & 100 & 0 & 0 & 45 & 0 & 75 & 0 & 100 & 100 & 12 & 0 & 0 & 40\end{array}$

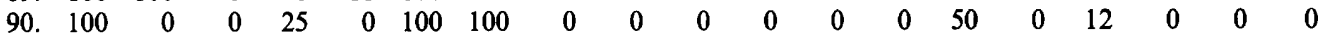
91. $100 \begin{array}{rllllllllllllllllll} & 50 & 0 & 25 & 11 & 100 & 100 & 0 & 60 & 0 & 33 & 25 & 0 & 100 & 78 & 0 & 80 & 0 & 20\end{array}$

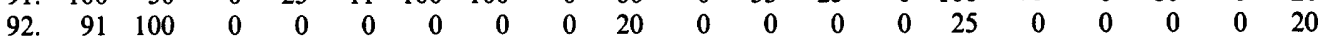
93. $100 \begin{array}{lllllllllllllllllll} & 100 & 50 & 100 & 0 & 100 & 100 & 0 & 0 & 45 & 17 & 100 & 0 & 100 & 100 & 12 & 0 & 50 & 60\end{array}$

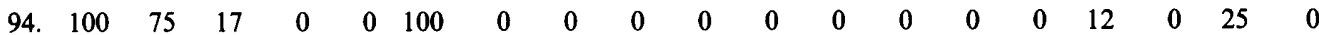

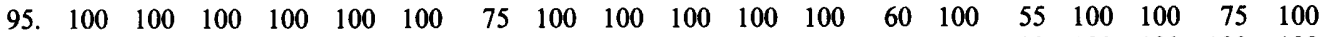

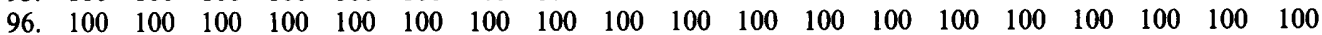

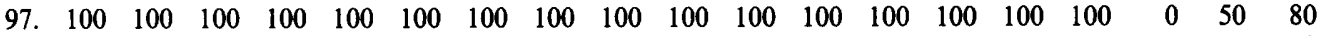

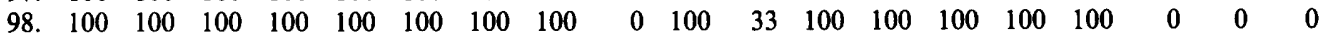

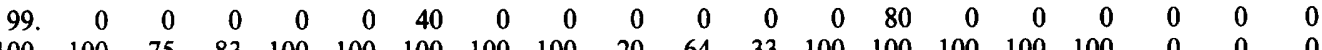
100. $100 \begin{array}{rllllllllllllllllll} & 75 & 83 & 100 & 100 & 100 & 100 & 100 & 20 & 64 & 33 & 100 & 100 & 100 & 100 & 100 & 0 & 0 & 0\end{array}$ 
Table 4 (continued)

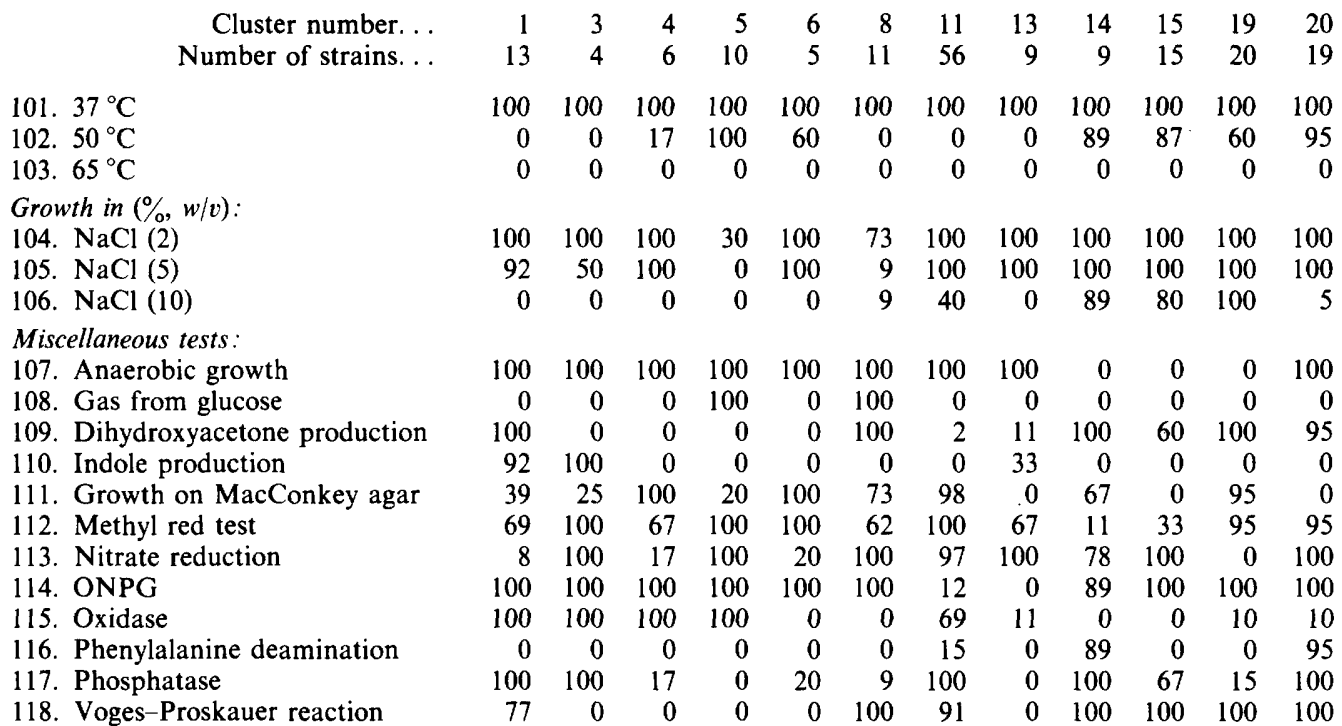

analysis of phenotypic features (Logan et al., 1979), although there are no clear diagnostic features. The results of the present study indicate that strains associated with incidents of food poisoning cannot be separated easily from other strains using phenotypic tests. Strains of ' $B$. cereus var. mycoides' were recovered in a separate subcluster (11I), and were distinguished by their characteristic colonial morphology.

B. psychrosaccharolyticus was recovered as a well-defined cluster in this study, a result in line with earlier work (Laine, 1970; Gyllenberg \& Laine, 1971). This species was not included in the Approved Lists of Bacterial Names (Skerman et al., 1980), but is listed as species incertae cedis in Bergey's Manual of Systematic Bacteriology (Claus \& Berkeley, 1986). It is evident from the present and earlier studies that the epithet $B$. psychrosaccharolyticus should be reintroduced (see below).

The ' $B$. subtilis group', defined at $78 \% S_{\mathrm{SM}}$, contained clusters identified as $B$. amyloliquefaciens, B. licheniformis, B. pumilus and B. subtilis. B. amyloliquefaciens and B. subtilis strains share little DNA sequence homology (Welker \& Campbell, 1967; Seki et al., 1975; Priest, 1981), can be separated by pyrolysis gas-liquid chromatography (O'Donnell et al., 1980) and can be distinguished by a few phenotypic properties (Priest et al., 1987). Our results support the recent demonstration that strains of B. amyloliquefaciens, unlike those of $B$. subtilis, produce acid from lactose (Nakamura, 1987), which is a useful distinguishing character.

The clear separation of $\boldsymbol{B}$. licheniformis, B. pumilis and $B$. subtilis has been noted in other taxometric studies (Bonde, 1975; Durand et al., 1979; O'Donnell et al., 1980; Logan \& Berkeley, 1981). These species comprise discrete DNA homology groups (reviewed by Priest, 1981) and can be readily distinguished by a number of presumptively diagnostic features (Table 4). It was also encouraging that strains labelled as ' $B$. aterrimus' and ' $B$. vulgatus' fell within the $B$. subtilis cluster, as such strains were recovered in the $B$. subtilis DNA homology group by Seki et al. (1975). Three strains of ' $B$. subtilis var. niger', were assigned to a separate cluster that was closely related to $B$. subtilis. However further studies are required to determine the taxonomic status of 'B. subtilis var. niger', as a strain of this taxon showed $95 \%$ DNA homology with the type strain of B. subtilis (Seki et al., 1975).

Strains labelled as $B$. megaterium were assigned to three DNA homology groups by Hunger \& Claus (1981). The homology group corresponding to $B$. megaterium sensu stricto is represented by cluster 22 . A second DNA homology group contains strains originally labelled as ' $B$. simplex' 


\begin{tabular}{|c|c|c|c|c|c|c|c|c|c|c|c|c|c|c|c|c|c|c|c|}
\hline & \multicolumn{19}{|c|}{ Table 4 (continued) } \\
\hline & 22 & 25 & 27 & 29 & 30 & 31 & 33 & 35 & 36 & 37 & 38 & 39 & 40 & 42 & 43 & 44 & 46 & 48 & 49 \\
\hline & 11 & 8 & 6 & 4 & 9 & 5 & 4 & 7 & 5 & 11 & 6 & 4 & 5 & 4 & 9 & 8 & 5 & 4 & 5 \\
\hline 101. & 100 & 100 & 100 & 100 & 100 & 100 & 100 & 100 & 100 & 100 & 100 & 100 & 0 & 100 & 100 & 100 & 100 & 75 & 100 \\
\hline 102. & 0 & 0 & 33 & 0 & 100 & 40 & 0 & 0 & 100 & 100 & 0 & 50 & 0 & 0 & 0 & 0 & 100 & 100 & 100 \\
\hline 103. & 0 & 0 & 0 & 0 & 0 & 0 & 0 & 0 & 0 & 0 & 0 & 0 & 0 & 0 & 0 & 0 & 0 & 100 & 100 \\
\hline 104. & 100 & 100 & 100 & 100 & 100 & 100 & 100 & 100 & 100 & 100 & 67 & 100 & 80 & 25 & 100 & 100 & 20 & 50 & 80 \\
\hline 105. & 100 & 100 & 100 & 100 & 100 & 100 & 100 & 100 & 0 & 100 & 33 & 100 & 80 & 25 & 89 & 100 & 0 & 25 & 60 \\
\hline 106. & 0 & 100 & 83 & 100 & 100 & 100 & 0 & 100 & 0 & 0 & 0 & 0 & 0 & 0 & 0 & 12 & 0 & 0 & 0 \\
\hline 107. & 0 & 0 & 0 & 0 & 100 & 0 & 0 & 0 & 0 & 0 & 0 & 0 & 0 & 0 & 0 & 0 & 100 & 50 & 0 \\
\hline 108. & 0 & 0 & 0 & 0 & 0 & 0 & 0 & 0 & 0 & 0 & 0 & 0 & 0 & 0 & 0 & 0 & 0 & 0 & 0 \\
\hline 109. & 0 & 0 & 0 & 0 & 0 & 0 & 0 & 0 & 0 & 0 & 0 & 0 & 0 & 0 & 11 & 0 & 0 & 0 & 0 \\
\hline 110. & 0 & 0 & 0 & 0 & 0 & 0 & 0 & 0 & 0 & 0 & 0 & 0 & 0 & 0 & 0 & 0 & 0 & 0 & 0 \\
\hline 111. & 100 & 63 & 100 & 75 & 100 & 100 & 100 & 14 & 0 & 36 & 33 & 100 & 100 & 100 & 100 & 100 & 20 & 25 & 60 \\
\hline 112 & 73 & 0 & 17 & 0 & 0 & 0 & 0 & 0 & 0 & 0 & 0 & 0 & 0 & 0 & 0 & 0 & 100 & 75 & 0 \\
\hline 113 & 0 & 100 & 0 & 0 & 89 & 100 & 0 & 0 & 100 & 91 & 100 & 50 & 80 & 0 & 44 & 37 & 100 & 100 & 100 \\
\hline 114 & 100 & 0 & 83 & 100 & 89 & 0 & 0 & 100 & 0 & 18 & 0 & 0 & 80 & 0 & 0 & 100 & 80 & 25 & 20 \\
\hline 115 & 0 & 0 & 17 & 0 & 22 & 0 & 0 & 0 & 60 & 0 & 83 & 25 & 20 & 50 & 89 & 100 & 0 & 0 & 0 \\
\hline 116 & 100 & 75 & 67 & 50 & 56 & 0 & 0 & 0 & 0 & 0 & 0 & 25 & 0 & 25 & 11 & 0 & 0 & 0 & 0 \\
\hline 117 & 54 & 0 & 17 & 0 & 0 & 20 & 0 & 0 & 80 & 0 & 0 & 0 & 0 & 50 & 33 & 0 & 80 & 25 & 100 \\
\hline 118 & 0 & 0 & 0 & 0 & 0 & 0 & 0 & 0 & 0 & 0 & 0 & 0 & 0 & 0 & 0 & 0 & 60 & 0 & 0 \\
\hline
\end{tabular}

and ' $B$. teres'. These strains are phenetically different from $B$. megaterium sensu stricto, and in the taxometric study of Priest $e t$ al. (1981) they were recovered in a different aggregate cluster from $B$. megaterium. In the present study, ' $B$. simplex' S210 and ' $B$. teres' $\mathbf{S} 213$ formed cluster 32 (cluster-group $C$ ), thereby supporting the distinction between these organisms and strains belonging to $B$. megaterium sensu stricto.

The third DNA homology group described by Hunger \& Claus (1981) encompassed strains originally labelled as ' $B$. agrestis' and ' $B$. flexus'. These organisms were recovered in a separate phenon (cluster 23) in both this and a previous study (Priest et al., 1981) and clearly represent a distinct species. Since the name ' $B$. flexus' (Batchelor, 1919) has priority over ' $B$. agrestis' (Werner, 1933) the former epithet is used for the reintroduced taxon (see below). Single-member clusters recovered in cluster-group B included strains of ' $B$. longissimus' (Mishustin \& Tepper, 1948) and ' $B$. maroccanus' (Delaporte \& Sasson, 1967). More strains of these taxa must be isolated and studied before their taxonomic status can be clarified.

Cluster-group $C$. Two groups of strains labelled as ' $B$. carotarum' and a third group associated with this name were recovered in cluster-group C. Strains S51 to S55 (cluster 31) were isolated by Gibson (1935) and identified by him as ' $B$. carotarum' sensu Koch 1888 . Two other strains originally labelled as ' $B$. carotarum' (NRS 608 and NRS 828) were donated by Gordon and thought to be original isolates from G. Bredemann and C. Stapp \& N. H. Claussen, respectively (R. E. Gordon, personal communication). These were recovered in cluster 33. The third group comprised strains originally labelled as 'B. simplex' and ' $B$. teres'. Such strains were assigned to DNA homology group B by Hunger \& Claus (1981) and were considered to belong to ' $\boldsymbol{B}$. carotarum' by Gibson \& Gordon (1974). These were recovered as cluster 32 . The fact that strains assigned to clusters 31,32 , and 33 have many properties in common helps to explain the confusion that has arisen with respect to the taxonomy of ' $B$. carotarum'. It is, however, clear from the present study that these taxa are distinct. The integrity of cluster 32 is supported by DNA base composition and reassociation data (Hunger \& Claus, 1981) and merits species status. Since these strains were not original isolates of Koch (1888), and to avoid confusion, they cannot be given the name ' $B$. carotarum'. The name ' $B$. simplex' (Gottheil, 1901) should be reintroduced for the taxon represented by cluster 32 as this epithet has priority over ' $B$. teres' (Neide, 1904). Further DNA studies are needed to confirm the taxonomic status of clusters 31 and 33 . 
Table 5. Distribution of positive characters to minor clusters defined at the $83 \%$ level $\left(S_{\mathrm{SM}}\right)$

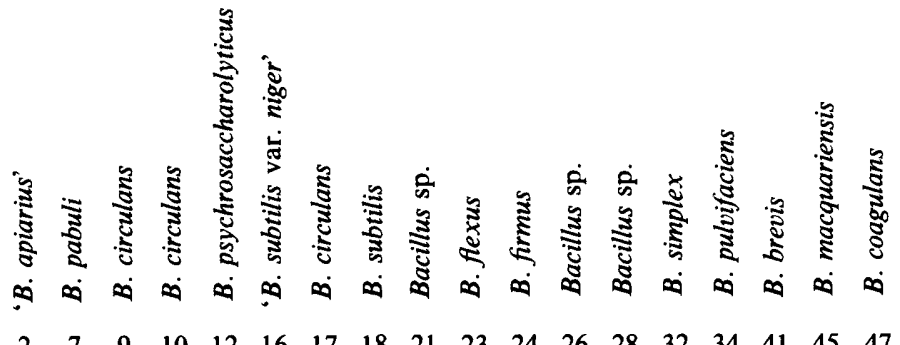

$\begin{array}{lllllllllllllllllll}\text { Cluster number... } & 2 & 7 & 9 & 10 & 12 & 16 & 17 & 18 & 21 & 23 & 24 & 26 & 28 & 32 & 34 & 41 & 45 & 47\end{array}$

$\begin{array}{lllllllllllllllllll}\text { Number of strains. .. } & 2 & 2 & 3 & 3 & 2 & 3 & 2 & 2 & 2 & 2 & 2 & 2 & 2 & 2 & 3 & 2 & 2 & 3\end{array}$

Colonial morphology
1. Flat/raised
2. Smooth
3. Rhizoidal
4. Entire
5. Opaque
6. Pigmented
7. Motile colonies

$\begin{array}{llllllllllllllllll}2 & 2 & 1 & 2 & 2 & 2 & 0 & 2 & 1 & 2 & 2 & 2 & 1 & 2 & 0 & 2 & 2 & 3 \\ 2 & 2 & 3 & 3 & 2 & 2 & 0 & 1 & 2 & 2 & 2 & 2 & 2 & 2 & 3 & 2 & 2 & 0 \\ 0 & 0 & 0 & 0 & 0 & 0 & 0 & 0 & 0 & 0 & 0 & 0 & 0 & 0 & 0 & 0 & 2 & 0 \\ 2 & 0 & 3 & 2 & 1 & 1 & 1 & 1 & 1 & 0 & 2 & 0 & 2 & 2 & 3 & 2 & 2 & 2 \\ 0 & 2 & 3 & 3 & 2 & 3 & 2 & 1 & 2 & 2 & 2 & 2 & 2 & 2 & 3 & 2 & 0 & 1 \\ 2 & 0 & 0 & 0 & 0 & 0 & 0 & 1 & 0 & 0 & 1 & 0 & 1 & 0 & 0 & 0 & 0 & 0 \\ 0 & 0 & 0 & 0 & 0 & 0 & 0 & 0 & 0 & 0 & 0 & 0 & 0 & 0 & 0 & 0 & 0 & 0\end{array}$

Cellular morphology

8. Length $>3 \mu \mathrm{m}$

9. Diameter $>0.9 \mu \mathrm{m}$

10. Ends round

11. Single

12. Vacuoles present

13. Gram-variable

14. Gram-positive

15. Spores oval

16. Spores round

17. Spores central

18. Spores terminal

19. Spores bulging

20. Sporulation $24 \mathrm{~h}$

21. Sporulation $72 \mathrm{~h}$

22. Sporulation $120 \mathrm{~h}$

23. Sporulation SxA

Degradation of:

24. Adenine

25. Aesculin

26. Allantoin

27. Arbutin

28. Casein

29. Chitin

30. DNA

31. Elastin

32. Gelatin

33. Guanine

34. Hippurate

35. Lecithin

36. Pectin

37. Pullulan

38. Pustulan

39. RNA

40. Starch

41. Testosterone

42. Tween 20

43. Tween 80

44. Tyrosine

45. Urea

$\begin{array}{llllllllllllllllll}2 & 3 & 3 & 3 & 0 & 0 & 1 & 0 & 0 & 1 & 0 & 2 & 2 & 0 & 0 & 1 & 1 & 3 \\ 0 & 0 & 0 & 0 & 2 & 0 & 0 & 0 & 0 & 1 & 0 & 0 & 1 & 0 & 0 & 0 & 0 & 0 \\ 0 & 2 & 3 & 3 & 2 & 3 & 2 & 2 & 2 & 2 & 2 & 2 & 2 & 2 & 2 & 2 & 2 & 3 \\ 2 & 2 & 3 & 3 & 2 & 0 & 2 & 2 & 2 & 0 & 2 & 0 & 0 & 0 & 0 & 2 & 0 & 2 \\ 0 & 0 & 0 & 0 & 0 & 0 & 0 & 0 & 0 & 1 & 0 & 0 & 0 & 0 & 0 & 0 & 0 & 0 \\ 0 & 0 & 0 & 0 & 2 & 3 & 1 & 2 & 2 & 2 & 2 & 2 & 2 & 1 & 2 & 2 & 0 & 2 \\ 0 & 0 & 0 & 0 & 0 & 1 & 0 & 1 & 2 & 0 & 0 & 2 & 2 & 0 & 0 & 0 & 0 & 0 \\ 2 & 2 & 3 & 3 & 2 & 3 & 2 & 2 & 1 & 2 & 2 & 2 & 2 & 2 & 3 & 2 & 2 & 3 \\ 0 & 0 & 0 & 0 & 0 & 0 & 0 & 0 & 1 & 0 & 0 & 0 & 0 & 0 & 0 & 0 & 0 & 0 \\ 2 & 1 & 0 & 1 & 2 & 3 & 2 & 2 & 1 & 2 & 2 & 2 & 2 & 2 & 3 & 0 & 2 & 2 \\ 0 & 1 & 3 & 2 & 0 & 2 & 0 & 0 & 1 & 0 & 0 & 0 & 0 & 0 & 0 & 2 & 0 & 1 \\ 2 & 2 & 3 & 3 & 2 & 0 & 1 & 0 & 2 & 0 & 0 & 0 & 0 & 0 & 3 & 2 & 2 & 1 \\ 0 & 1 & 0 & 1 & 0 & 2 & 2 & 0 & 1 & 2 & 1 & 0 & 0 & 0 & 0 & 2 & 0 & 3 \\ 1 & 2 & 2 & 3 & 1 & 3 & 2 & 0 & 2 & 2 & 2 & 2 & 2 & 2 & 1 & 2 & 0 & 3 \\ 2 & 2 & 3 & 3 & 2 & 3 & 2 & 2 & 2 & 2 & 2 & 2 & 2 & 2 & 3 & 2 & 0 & 3 \\ 2 & 2 & 3 & 3 & 2 & 3 & 2 & 2 & 2 & 2 & 2 & 2 & 2 & 2 & 3 & 2 & 2 & 3\end{array}$

$\begin{array}{llllllllllllllllll}0 & 0 & 0 & 0 & 0 & 1 & 0 & 1 & 0 & 0 & 0 & 0 & 0 & 0 & 0 & 2 & 0 & 3\end{array}$ $\begin{array}{llllllllllllllllll}2 & 2 & 3 & 3 & 2 & 3 & 2 & 2 & 2 & 0 & 2 & 0 & 2 & 0 & 0 & 0 & 2 & 2\end{array}$ $\begin{array}{llllllllllllllllll}0 & 0 & 0 & 0 & 2 & 0 & 0 & 0 & 2 & 0 & 0 & 0 & 0 & 0 & 0 & 2 & 0 & 0\end{array}$ $\begin{array}{llllllllllllllllll}2 & 2 & 3 & 3 & 2 & 3 & 2 & 2 & 2 & 2 & 2 & 1 & 2 & 2 & 0 & 0 & 2 & 3\end{array}$ $\begin{array}{llllllllllllllllll}2 & 2 & 2 & 1 & 2 & 3 & 2 & 2 & 2 & 2 & 2 & 2 & 2 & 2 & 3 & 2 & 0 & 3\end{array}$ $\begin{array}{llllllllllllllllll}0 & 0 & 0 & 0 & 0 & 2 & 0 & 0 & 2 & 0 & 0 & 0 & 0 & 0 & 0 & 0 & 0 & 0\end{array}$ $\begin{array}{llllllllllllllllll}2 & 2 & 0 & 2 & 2 & 3 & 2 & 2 & 2 & 2 & 2 & 2 & 2 & 1 & 0 & 2 & 0 & 3\end{array}$ $\begin{array}{llllllllllllllllll}0 & 0 & 0 & 0 & 2 & 1 & 2 & 2 & 1 & 2 & 2 & 0 & 0 & 0 & 0 & 2 & 0 & 0\end{array}$ $\begin{array}{llllllllllllllllll}2 & 2 & 0 & 2 & 2 & 3 & 2 & 2 & 2 & 2 & 2 & 2 & 2 & 2 & 3 & 2 & 0 & 3\end{array}$ $\begin{array}{llllllllllllllllll}0 & 0 & 0 & 0 & 0 & 0 & 0 & 0 & 0 & 0 & 0 & 1 & 0 & 0 & 0 & 0 & 0 & 0\end{array}$ $\begin{array}{llllllllllllllllll}2 & 0 & 1 & 3 & 0 & 0 & 1 & 0 & 0 & 0 & 0 & 1 & 0 & 2 & 1 & 0 & 0 & 3\end{array}$ $\begin{array}{llllllllllllllllll}2 & 0 & 0 & 0 & 2 & 3 & 2 & 1 & 2 & 0 & 1 & 2 & 1 & 0 & 0 & 0 & 0 & 0\end{array}$ $\begin{array}{llllllllllllllllll}0 & 2 & 0 & 0 & 0 & 1 & 0 & 2 & 2 & 0 & 0 & 0 & 0 & 0 & 2 & 0 & 0 & 0\end{array}$ $\begin{array}{llllllllllllllllll}2 & 0 & 3 & 0 & 2 & 2 & 0 & 1 & 0 & 2 & 1 & 2 & 2 & 0 & 1 & 0 & 2 & 0\end{array}$ $\begin{array}{llllllllllllllllll}0 & 2 & 0 & 1 & 0 & 0 & 0 & 0 & 0 & 0 & 0 & 0 & 0 & 0 & 0 & 0 & 0 & 0\end{array}$ $\begin{array}{llllllllllllllllll}2 & 2 & 3 & 1 & 2 & 3 & 2 & 2 & 2 & 2 & 2 & 2 & 2 & 1 & 3 & 2 & 0 & 3\end{array}$ $\begin{array}{llllllllllllllllll}2 & 2 & 3 & 2 & 2 & 3 & 2 & 2 & 2 & 2 & 1 & 2 & 1 & 2 & 0 & 0 & 2 & 3\end{array}$ $\begin{array}{llllllllllllllllll}0 & 0 & 3 & 1 & 0 & 0 & 0 & 0 & 0 & 0 & 1 & 0 & 0 & 0 & 0 & 2 & 0 & 0\end{array}$ $\begin{array}{llllllllllllllllll}2 & 2 & 3 & 3 & 2 & 3 & 2 & 2 & 2 & 2 & 0 & 2 & 2 & 2 & 3 & 2 & 0 & 3\end{array}$ $\begin{array}{lllllllllllllllllll}2 & 2 & 3 & 3 & 2 & 3 & 2 & 1 & 2 & 1 & 1 & 2 & 1 & 1 & 2 & 2 & 0 & 3\end{array}$ $\begin{array}{llllllllllllllllll}2 & 0 & 0 & 0 & 0 & 0 & 0 & 0 & 0 & 0 & 0 & 0 & 0 & 2 & 0 & 0 & 0 & 0\end{array}$ 
Table 5 (continued)

$\begin{array}{lllllllllllllllllll}\text { Cluster number... } & 2 & 7 & 9 & 10 & 12 & 16 & 17 & 18 & 21 & 23 & 24 & 26 & 28 & 32 & 34 & 41 & 45 & 47\end{array}$ $\begin{array}{lllllllllllllllllll}\text { Number of strains. .. } & 2 & 2 & 3 & 3 & 2 & 3 & 2 & 2 & 2 & 2 & 2 & 2 & 2 & 2 & 3 & 2 & 2 & 3\end{array}$

Resistance to $\left(\mu \mathrm{g} \mathrm{ml}^{-1}\right)$ :

46. Benzylpenicillin (8)

47. Benzylpenicillin (4)

48. Chloramphenicol (8)

49. Chloramphenicol (4)

50. Cycloserine (128)

51. Cycloserine (64)

52. Erythromycin (1)

53. Erythromycin $(0 \cdot 5)$

54. Gramicidin (64)

55. Gramicidin (32)

56. Nalidixic acid (32)

57. Nalidixic acid (16)

58. Polymyxin (16)

59. Polymyxin (8)

60. Rifampicin $(0 \cdot 25)$

61. Rifampicin $(0 \cdot 125)$

62. Streptomycin (16)

63. Streptomycin (8)

64. Tetracycline (2)

65. Tetracycline (1)

$\begin{array}{llllllllllllllllll}0 & 0 & 0 & 0 & 1 & 3 & 0 & 0 & 0 & 0 & 0 & 2 & 0 & 0 & 0 & 0 & 0 & 0 \\ 0 & 0 & 0 & 0 & 1 & 3 & 1 & 0 & 0 & 0 & 0 & 2 & 0 & 0 & 0 & 0 & 0 & 0 \\ 0 & 2 & 0 & 0 & 1 & 1 & 0 & 0 & 2 & 0 & 0 & 2 & 0 & 0 & 0 & 2 & 0 & 0 \\ 0 & 2 & 1 & 0 & 1 & 2 & 0 & 0 & 2 & 0 & 0 & 2 & 0 & 2 & 0 & 2 & 0 & 0 \\ 0 & 2 & 3 & 0 & 1 & 2 & 1 & 0 & 0 & 0 & 0 & 0 & 0 & 0 & 1 & 2 & 2 & 0 \\ 2 & 2 & 3 & 0 & 1 & 3 & 2 & 2 & 0 & 0 & 0 & 1 & 1 & 1 & 3 & 2 & 2 & 0 \\ 0 & 2 & 0 & 0 & 0 & 0 & 0 & 0 & 2 & 1 & 0 & 2 & 0 & 0 & 0 & 2 & 0 & 0 \\ 0 & 2 & 0 & 0 & 0 & 0 & 0 & 0 & 2 & 2 & 0 & 2 & 0 & 0 & 0 & 2 & 0 & 0 \\ 2 & 0 & 0 & 0 & 1 & 3 & 2 & 2 & 2 & 1 & 2 & 2 & 0 & 0 & 1 & 2 & 0 & 0 \\ 2 & 0 & 0 & 1 & 2 & 3 & 2 & 2 & 2 & 1 & 2 & 2 & 0 & 0 & 1 & 2 & 0 & 0 \\ 0 & 0 & 3 & 1 & 0 & 0 & 0 & 0 & 2 & 0 & 0 & 0 & 0 & 2 & 0 & 2 & 1 & 3 \\ 0 & 0 & 3 & 1 & 0 & 0 & 0 & 0 & 2 & 0 & 0 & 0 & 0 & 2 & 3 & 2 & 2 & 3 \\ 2 & 2 & 0 & 0 & 0 & 3 & 2 & 2 & 2 & 0 & 0 & 0 & 0 & 0 & 3 & 2 & 0 & 0 \\ 2 & 2 & 1 & 0 & 0 & 3 & 2 & 2 & 2 & 0 & 0 & 0 & 0 & 1 & 3 & 2 & 1 & 0 \\ 0 & 1 & 0 & 0 & 0 & 0 & 2 & 1 & 0 & 0 & 0 & 2 & 0 & 1 & 0 & 1 & 0 & 0 \\ 0 & 1 & 0 & 0 & 1 & 1 & 2 & 2 & 2 & 1 & 2 & 2 & 0 & 1 & 0 & 1 & 0 & 0 \\ 2 & 2 & 0 & 0 & 0 & 1 & 0 & 2 & 2 & 0 & 0 & 0 & 0 & 0 & 0 & 2 & 2 & 0 \\ 2 & 2 & 0 & 0 & 1 & 2 & 0 & 2 & 2 & 0 & 0 & 0 & 0 & 0 & 2 & 2 & 2 & 0 \\ 0 & 0 & 0 & 0 & 1 & 0 & 2 & 2 & 0 & 0 & 0 & 0 & 0 & 0 & 0 & 0 & 0 & 0 \\ 2 & 0 & 0 & 0 & 1 & 3 & 2 & 2 & 2 & 0 & 1 & 0 & 0 & 0 & 0 & 0 & 0 & 0\end{array}$

Acid from:

66. Adonitol

67. Arabinose

68. Cellobiose

69. Dulcitol

70. Erythritol

71. Fructose

72. Galactose

73. Glucose

74. Glycerol

75. meso-Inositol

76. Lactose

77. Maltose

78. Mannitol

79. Mannose

80. Raffinose

81. Rhamnose

82. Salicin

83. Sorbitol

84. Sucrose

85. Trehalose

86. Xylose

$\begin{array}{llllllllllllllllll}0 & 0 & 0 & 1 & 0 & 0 & 0 & 0 & 0 & 0 & 0 & 0 & 0 & 0 & 0 & 0 & 0 & 0 \\ 0 & 0 & 0 & 0 & 0 & 0 & 1 & 0 & 0 & 0 & 0 & 0 & 0 & 0 & 0 & 0 & 0 & 0 \\ 2 & 2 & 3 & 2 & 1 & 3 & 2 & 0 & 2 & 0 & 1 & 0 & 0 & 2 & 0 & 0 & 2 & 0 \\ 0 & 0 & 0 & 0 & 0 & 0 & 0 & 0 & 0 & 0 & 0 & 0 & 0 & 0 & 0 & 0 & 0 & 0 \\ 0 & 0 & 0 & 0 & 0 & 0 & 0 & 0 & 0 & 0 & 2 & 1 & 0 & 2 & 3 & 0 & 0 & 3 \\ 0 & 2 & 3 & 3 & 2 & 3 & 2 & 2 & 2 & 2 & 2 & 0 & 0 & 0 & 0 & 0 & 2 & 0 \\ 2 & 1 & 3 & 3 & 0 & 3 & 0 & 0 & 2 & 2 & 2 & 1 & 0 & 2 & 3 & 0 & 2 & 3 \\ 2 & 2 & 3 & 3 & 2 & 3 & 2 & 2 & 2 & 2 & 2 & 1 & 0 & 2 & 3 & 0 & 2 & 3 \\ 2 & 2 & 2 & 1 & 2 & 3 & 2 & 0 & 2 & 2 & 2 & 2 & 2 & 2 & 2 & 2 & 0 & 2 \\ 2 & 1 & 0 & 0 & 0 & 3 & 2 & 0 & 2 & 1 & 0 & 0 & 0 & 1 & 0 & 0 & 1 & 0 \\ 0 & 2 & 3 & 3 & 0 & 1 & 1 & 0 & 0 & 2 & 0 & 0 & 0 & 0 & 3 & 0 & 0 & 0 \\ 2 & 2 & 3 & 3 & 2 & 3 & 1 & 2 & 2 & 2 & 2 & 1 & 1 & 2 & 3 & 0 & 1 & 3 \\ 0 & 2 & 3 & 0 & 1 & 3 & 2 & 1 & 2 & 2 & 2 & 0 & 0 & 1 & 3 & 0 & 2 & 3 \\ 0 & 2 & 1 & 1 & 2 & 3 & 1 & 0 & 2 & 0 & 2 & 0 & 0 & 1 & 0 & 0 & 0 & 3 \\ 2 & 2 & 3 & 3 & 0 & 1 & 2 & 0 & 2 & 2 & 2 & 0 & 0 & 0 & 0 & 0 & 2 & 0 \\ 0 & 0 & 0 & 0 & 0 & 0 & 0 & 0 & 1 & 0 & 0 & 0 & 0 & 0 & 0 & 0 & 0 & 0 \\ 2 & 2 & 3 & 2 & 1 & 3 & 2 & 0 & 2 & 1 & 0 & 0 & 0 & 0 & 0 & 0 & 0 & 0 \\ 0 & 1 & 0 & 0 & 1 & 3 & 2 & 1 & 2 & 0 & 0 & 0 & 0 & 0 & 0 & 0 & 0 & 0 \\ 2 & 2 & 3 & 3 & 1 & 3 & 2 & 2 & 2 & 2 & 2 & 1 & 0 & 2 & 0 & 0 & 1 & 3 \\ 2 & 2 & 3 & 3 & 2 & 3 & 2 & 2 & 2 & 2 & 2 & 2 & 1 & 2 & 3 & 0 & 0 & 3 \\ 0 & 2 & 3 & 1 & 2 & 3 & 1 & 0 & 2 & 0 & 0 & 0 & 0 & 0 & 0 & 0 & 2 & 0\end{array}$

Utilization of:

87. Acetate

88. Citrate

89. Formate

90. Gluconate

91. Lactate

92. Malonate

93. Succinate

$\begin{array}{llllllllllllllllll}1 & 0 & 1 & 0 & 0 & 3 & 2 & 0 & 2 & 2 & 1 & 1 & 0 & 2 & 2 & 2 & 0 & 0 \\ 0 & 0 & 0 & 0 & 0 & 3 & 1 & 0 & 2 & 2 & 1 & 2 & 0 & 2 & 3 & 0 & 0 & 3 \\ 0 & 0 & 0 & 1 & 1 & 3 & 2 & 1 & 0 & 2 & 2 & 2 & 0 & 2 & 1 & 2 & 1 & 1 \\ 0 & 1 & 1 & 3 & 0 & 0 & 0 & 0 & 1 & 0 & 0 & 3 & 1 & 1 & 0 & 0 & 2 & 1 \\ 0 & 0 & 0 & 3 & 1 & 3 & 0 & 0 & 0 & 0 & 0 & 0 & 0 & 1 & 0 & 0 & 2 & 1 \\ 0 & 0 & 0 & 0 & 0 & 0 & 0 & 0 & 0 & 0 & 0 & 2 & 1 & 0 & 0 & 0 & 0 & 0 \\ 0 & 0 & 3 & 2 & 0 & 3 & 1 & 2 & 2 & 2 & 2 & 2 & 0 & 2 & 3 & 1 & 0 & 0\end{array}$

Growth at :

94. $\mathrm{pH} 4.5$

95. $\mathrm{pH} 6.0$

96. $\mathrm{pH} 7 \cdot 2$

97. $\mathrm{pH} 8.0$

98. $\mathrm{pH} 9 \cdot 5$

99. $5^{\circ} \mathrm{C}$

$\begin{array}{llllllllllllllllll}0 & 0 & 0 & 0 & 0 & 0 & 0 & 1 & 0 & 2 & 0 & 0 & 0 & 0 & 0 & 0 & 0 & 1 \\ 2 & 2 & 3 & 3 & 1 & 3 & 2 & 2 & 2 & 2 & 2 & 2 & 2 & 2 & 3 & 2 & 2 & 3 \\ 2 & 2 & 3 & 3 & 2 & 3 & 2 & 2 & 2 & 2 & 2 & 2 & 2 & 2 & 3 & 2 & 2 & 0 \\ 2 & 2 & 3 & 3 & 2 & 3 & 2 & 2 & 2 & 2 & 2 & 2 & 2 & 2 & 3 & 2 & 2 & 0 \\ 0 & 2 & 3 & 3 & 2 & 3 & 2 & 2 & 2 & 2 & 2 & 2 & 2 & 2 & 3 & 2 & 2 & 0 \\ 0 & 2 & 1 & 2 & 2 & 0 & 1 & 2 & 0 & 0 & 0 & 0 & 0 & 0 & 0 & 0 & 2 & 0\end{array}$




\section{Table 5 (continued)}

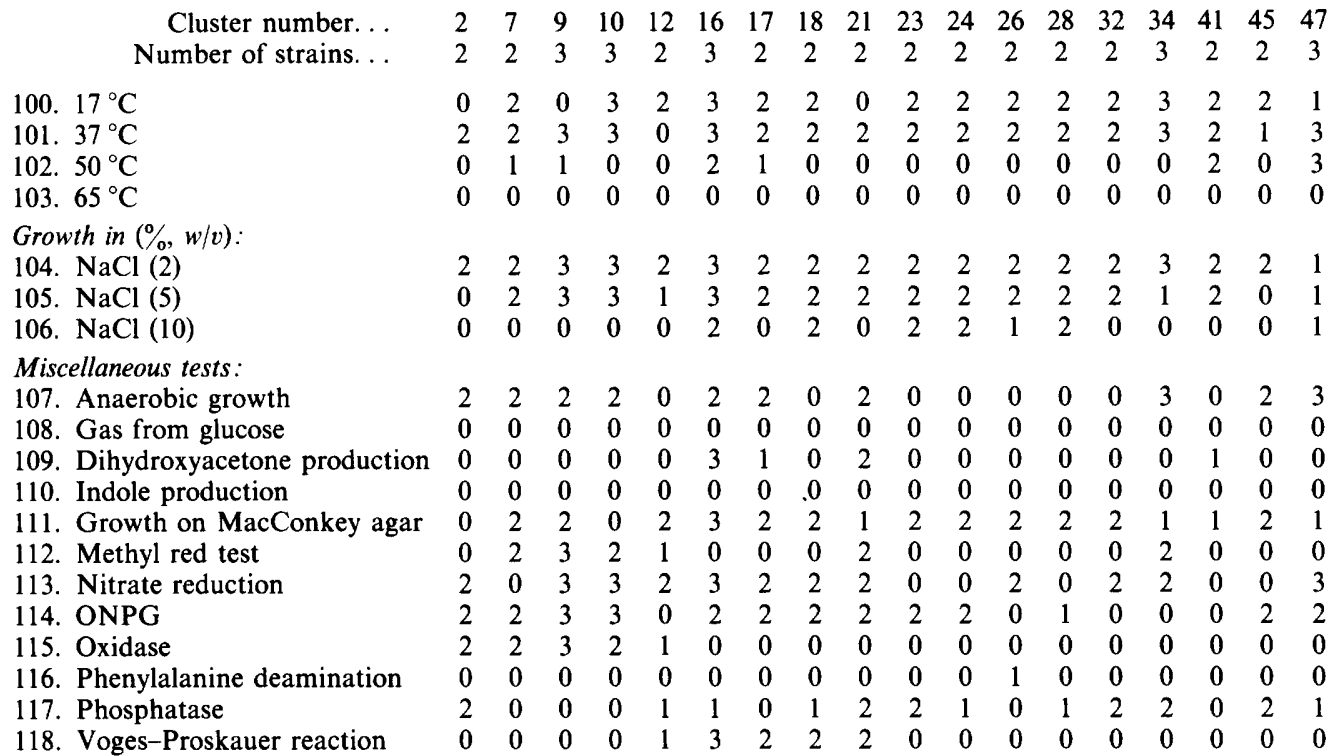

Cluster-group $\mathrm{C}$ also contained isolates from marine or saline environments. In a numerical taxonomic study of 138 bacilli isolated from the North Sea, Boeye \& Aerts (1976) recognized two major clusters representing $B$. firmus and the $B$. subtilis group. Similarly, Bonde $(1975,1976)$ examined several hundred bacilli from marine sources and found that $B$. firmus, species of the ' $B$. subtilis group', and $B$. sphaericus were common. In the present investigation, most of the representatives from the studies of Bonde $(1975,1976)$ and Boeyé \& Aerts $(1976)$ were assigned to cluster-groups $\mathrm{B}$ and $\mathrm{C}$.

B. firmus has been a problematical taxon (Gordon et al., 1977). In the present study, the $B$. firmus strains formed a compact phenon, a result in agreement with earlier work (Priest et al., 1981; Logan \& Berkeley, 1981). B. firmus was also distinguished from B. lentus (cluster 44) in DNA pairing experiments (Priest, 1981; Seki et al., 1983) which showed that the sequence homology between representatives of these species was very low. Many bacilli isolated from saline environments have been described as intermediate between $B$. firmus and B. lentus and, as a result, strains in these taxa have been considered to form a 'spectrum' (Gordon et al., 1977). In the present numerical classification, however, most of the $B$. firmus/B. lentus intermediates were recovered in three related but distinct phena, clusters 27,28 and 29 , within cluster-group $C$. These findings are supported by DNA reassociation data which indicate that very little homology exists between 'intermediate strains' and B. firmus (Priest, 1981; Seki et al., 1983). Strains NRS 1575 and NRS 1570, for example, not only belong to different clusters but have been assigned to distinct DNA homology groups (Seki et al., 1983). Similarly, strain NRS 1151 was assigned to an individual homology group and was recovered in cluster 26 . Further nucleic acid reassociation data are needed to resolve the taxonomic status of clusters 27,28 and 29.

Other phena that comprised distinct taxa within cluster-group C include $B$.pantothenticus and B. pulvifaciens. Strains received as 'Krusella cascainensis' (Castellani, 1954) produce ellipsoidal spores (Castellani, 1955; Gordon et al., 1973) and have been transferred to the genus Bacillus as ' $B$. cascainensis' (Castellani, 1955). The present study indicates that this epithet should be reintroduced, but DNA base composition data on representative strains are needed to complement the present description of this taxon. A single strain of 'B. epiphytus' was recovered on the periphery of the $B$. firmus cluster; this relationship has been noted by others (Gibson \& 
Gordon, 1974; Bonde, 1976; Logan \& Berkeley, 1981). DNA data are required to clarify the status of ' $B$. epiphytus'. Similarly, ' $B$. loehnisii', ' $B$. pacificus' and ' $B$. macroides' formed singlemember clusters in cluster-group C. 'Bacillus loehnisii' is generally regarded to be similar to $B$. pasteurii and ' $B$. freudenreichii' (Gibson, 1934) but in the present study, $B$. pasteurii strains were not included and ' $B$. freundenreichii' was recovered in cluster group D. 'Bacillus macroides', on the other hand, was assigned to the B. firmus aggregate group by Logan \& Berkeley (1981); its placement in cluster-group $\mathrm{C}$ is consistent with this.

Cluster-group D. Most of the B. brevis strains were recovered in cluster 37, but the allocation of two strains to cluster 41 was in good agreement with an earlier taxometric study where $B$. brevis was shown to be heterogeneous (Priest et al., 1981). Five strains of ' $B$. aneurinolyticus' formed a homogeneous phenon closely related to $B$. brevis. Previously, casein hydrolysis was considered the only feature available to distinguish between these taxa (Claus \& Berkeley, 1986), but additional differential characteristics have been highlighted in this study. The name ' $B$. aneurinolyticus' should be reintroduced when confirmatory DNA base composition data become available.

$B$. sphaericus strains have been assigned to at least five DNA homology groups (Seki et al., 1978; Krych et al., 1980), but still appear to be phenotypically uniform. Previous taxometric studies have placed B. sphaericus in a single phenon (Logan \& Berkeley, 1981; Priest et al., 1981) but in the present analysis four strains labelled ' $B$. sphaericus var. fusiformis' were assigned to a separate cluster. This cluster corresponds to DNA homology group IIB of Krych et al. (1980). It is evident that the strains of cluster 41 merit species status given the good congruence between the DNA homology and numerical phenetic data. The name $B$. fusiformis has been proposed for this taxon (see below). A strain of ' $B$. rotans' was assigned to DNA homology group III by Krych et al. (1980). The recovery of ' $B$. sphaericus var. rotans' (NCIB 8867) as a single-member cluster is in support with the view that this organism may also represent a new taxospecies.

The integrity of $B$. azotoformans (Pichinoty et al., 1983) was supported by the assignment of six representatives of this species to cluster 38 . A few psychrophilic strains were also recovered in cluster-group D. The numerical phenetic data support the current taxonomic status of $B$. globisporus, B. insolitus and B. psychrophilus (Larkin \& Stokes, 1967; Ruger, 1983; Nakamura, $1984 b$ ).

Cluster-group $E$. The clear separation of $B$. lentus (cluster 44) from $B$. firmus (cluster 25, clustergroup C) confirms the independent status of these species. The recovery of two strains of $B$. macquariensis in cluster-group $E$ casts doubt on the reported affinity between $B$. circulans and $B$. macquariensis (Gibson \& Gordon, 1974; Logan \& Berkeley, 1981).

Cluster-group $F$. The recovery of the $B$. coagulans and $B$. stearothermophilus strains in a single aggregate group is in good agreement with the earlier study of Logan \& Berkeley (1981) which showed that these bacteria have many features in common beyond their ability to grow at high temperature. B. coagulans comprises at least two phenetic groups (Wolf \& Barker, 1968), and although limited DNA reassociation studies indicated genetic homology (Seki et al., 1978), two DNA homology groups have subsequently been revealed (I. Blumenstock, personal communication: quoted by Claus \& Berkeley, 1986). In the present study, strains of $B$. coagulans were similarly assigned to two clusters, cluster 46 representing $B$. coagulans sensu stricto.

The recovery of the $B$. stearothermophilus strains in two major clusters and one single-member cluster provides yet further evidence for the heterogeneity of this taxon. It is generally accepted that $B$. stearothermophilus encompasses at least three distinct taxa (Baillie \& Walker, 1968; Klaushofer \& Hollaus, 1970; Walker \& Wolf, 1971; Sharp et al., 1980). Cluster 48 contained strains of B. stearothermophilus sensu stricto (Walker \& Wolf, 1971; group 3) although it also encompassed strains assigned by these workers to their group 2. Cluster 49 , which was particularly well defined, corresponds to group 1 ('B. kaustophilus') of Walker \& Wolf (1971). This taxon is phenetically and genotypically distinct from B. stearothermophilus (Sharp et al., 1980) and merits species status (see below). 


\section{The genus Bacillus, the emerging taxonomy}

It is appropriate in a wide-ranging study such as the present one, to draw some general conclusions and suggest priorities for the future. It is now evident that the genus Bacillus encompasses some 80 taxa of approximate species rank that can be assigned to five or more cluster-groups. The latter should be used as a framework for redefining the current genus and splitting it into several genera. An indication of how this might best be achieved has been revealed by Stackebrandt et al. (1987), who have shown that B. sphaericus and other species containing round-spored organisms can be distinguished from other bacilli on the basis of rRNA oligonucleotide sequencing, spore morphology and cell-wall composition studies. However, we agree with these authors that many more strains need to be studied by similar techniques before 'a formal dissection of the genus Bacillus with consequent description of new genera is proposed'.

It is also evident from the present study that several clusters merit species status given the appropriate supporting data from the literature, and formal proposals are given below. It is also highly likely that taxa such as ' $B$. aneurinolyticus', ' $B$. apiarius', ' $B$. cascainensis', ' $B$. thiaminolyticus' and the various halotolerant isolates described as ' $B$. firmus $-B$. lentus intermediates' should be raised to valid species status. Supporting DNA base composition and reassociation data are required before this can be recommended.

Further comparative studies are needed to revise and clarify the classification of heterogeneous species such as $B$. brevis, $B$. circulans, $B$. coagulans, $B$. sphaericus and $B$. stearothermophilus. It is also possible that strains carrying names such as ' $B$. cirroflagellosus', ' $B$. epiphytus', 'B. filicolonicus', ' $B$. freudenreichii', 'B. globigii', ' $B$. loehnisii', ' $B$. longissimus', ' $B$. macroides', ' $B$. maroccanus', ' $B$. pacificus' and ' $B$. repens' represent new centres of variation, but additional representatives of these taxa need to be examined to determine their taxonomic status.

\section{NOMENCLATURE}

Description of Bacillus flexus (Batchelor, 1919) nom. rev.

flex'us. L. adj. flexus, flexible.

The description given below is taken from the present and earlier studies (Hunger \& Claus, 1981 ; Claus \& Berkeley, 1986). Strains in this species have similar properties to B. megaterium but differ from typical members of that species as cells are smaller (mean cell width $0.9 \mu \mathrm{m}$ ), poly- $\beta$-hydroxybutyrate is not formed, phenylalanine is not deaminated, neither is aesculin hydrolysed nor acid formed from pentoses. Strains of this species degrade casein, elastin, gelatin, pullulan and starch, are urease positive, but give a negative Voges-Proskauer reaction and do not reduce nitrate to nitrite. Additional properties are given in Table 5.

The $\mathrm{mol} \% \mathrm{G}+\mathrm{C}$ content of the DNA of the two strains examined lies between 37 and 39 $\left(T_{\mathrm{m}}\right)$. The type strain has little in common with either ' $B$. carotarum' or $B$. megaterium.

Source: Faeces and soil.

Type strain: DSM 1320 (= NRS 665).

Description of Bacillus fusiformis (Smith et al., 1946) comb. nov. (Bacillus sphaericus var. fusiformis Smith, Gordon \& Clark, 1946, 97)

fus.i.form'is. L. n. fusus spindle; L. n. forma shape, form; M.L. adj. fusiformis spindle-shaped.

The description is taken from the present study and from that of Krych et al. (1980). Strains in this species have similar properties to $B$. sphaericus but differ from typical members of that species as they are urease positive, grow in the presence of $\mathrm{NaCl}(7 \%, \mathrm{w} / \mathrm{v})$ and are sensitive to tetracycline $\left(1 \mu \mathrm{g} \mathrm{ml}^{-1}\right)$. They are oxidase positive, degrade gelatin and testosterone, but give a negative Voges-Proskauer reaction, and do not degrade starch or reduce nitrate to nitrite. Additional properties are given in Table 4.

The $\mathrm{mol} \% \mathrm{G}+\mathrm{C}$ of the DNA falls within the range 35 to $36\left(T_{\mathrm{m}}\right)$ for the eleven strains examined. These strains form a distinct DNA homology group that is related to a second homology group which accommodates strains pathogenic for mosquitoes (Krych et al., 1980).

Source: Soil.

Type strain: ATCC 7055. 
Description of Bacillus kaustophilus (Prickett, 1928) nom. rev.

kau.sto.ph'il.us. Gr. n. kaustos, heat; Gr, adj. philus loving; M.L. adj. kaustophilus heat loving.

The description is taken from the present and several other studies (Prickett, 1928; Walker \& Wolf, 1971; Sharp et al., 1980). Strains in this species have similar properties to $B$. stearothermophilus but differ from members of this species by their ability to produce acid from cellobiose, meso-inositol and xylose, to degrade testosterone and to reduce nitrate to gas, and by their relative sensitivity to $\mathrm{NaCl}$ and failure to grow anaerobically. They produce oval to cylindrical spores that distend the sporangium to a greater or less extent, liquefy gelatin, degrade aesculin, arbutin, pullulan and starch (weakly), and grow optimally between 60 and $65^{\circ} \mathrm{C}$. Additional properties are given in Table 4.

The $\mathrm{mol} \% \mathrm{G}+\mathrm{C}$ of the DNA of the five strains studied falls within the range 51 to $55\left(T_{\mathrm{m}}\right)$. There is evidence that these strains form a distinct DNA homology group (Sharp et al., 1980).

Source: Pasteurized milk, deteriorated canned food and probably soil.

Type strain: ATCC 8005 (=N. R. Smith T281).

Description of Bacillus psychrosaccharolyticus (Larkin \& Stokes, 1967) nom. rev.

psy.chro.sac.char.o.lyt'i.cus. Gr. adj. psychros cold; Gr. n. saccharon sugar; Gr. adj. lytos dissolvable; M.L. adj. psychrosaccharolyticus cold (adapted), sugar-fermenting.

The description is taken from the present and two other studies (Larkins \& Stokes, 1967; Claus \& Berkeley, 1986). Cells are distinctly pleomorphic, varying from coccoid to elongate. On glucose media they may contain globules that are unstainable with fuchsin. Growth and sporulation occur at $0^{\circ} \mathrm{C}$. If sporulation does not occur, the organism may swell and become faintly stainable, of ten forming pear-shaped bodies up to $2 \mu \mathrm{m}$ in diameter. The spore frequently fills most of the sporangium; it may occur in a lateral position. Relatively thick opaque growth without spreading or outgrowths occurs on agar media. Overgrowth of laboratory cultures by asporogenous mutants appears to occur frequently. Glucose promotes anaerobic growth only slightly. Aesculin, allantoin and arbutin are hydrolysed, and elastin, gelatin, lecithin, pullulan and starch are degraded.

The $\mathrm{mol} \% \mathrm{G}+\mathrm{C}$ of the DNA lies within the range 43 to $44\left(T_{\mathrm{m}} ;\right.$ F.G. Priest, unpublished data).

Source: Soil and marshes.

Type strain: NCIB 11729 (=ATCC 23296 = DSM 6).

Direct plating of soil frequently yields organisms which have the characteristics of $B$. psychrosaccharolyticus except that some of them may diverge from that species in their action on nitrate (none or denitrification), proteins, starch, particular sugars, or in utilization of glucose for anaerobic growth. These organisms, which do not appear to have been named, have yet to be the subject of comparative studies to determine their possible relationship to $B$. psychrosaccharolyticus.

Description of Bacillus simplex (Gottheil, 1901) nom. rev.

sim'plex. L. adj. simplex simple.

The description is taken from the present study and that of Hunger \& Claus (1981). Strains in this species have properties in common with $B$. megaterium but differ from typical members of that species as they reduce nitrate to nitrite, produce brownish colonies on tyrosine agar, fail to hydrolyse aesculin and urea, do not deaminate phenylalanine or form hydroxybutyrate and have cells that measure only 0.8 to $1.0 \mu \mathrm{m}$ in diameter (a few broader cells are occasionally observed). They degrade arbutin, gelatin, starch and tyrosine but not chitin. They are negative for the Voges-Proskauer and egg-yolk tests and do not grow in the presence of lysozyme. Additional properties are given in Table 5 .

The $\mathrm{mol} \% \mathrm{G}+\mathrm{C}$ content of the DNA of the six strains examined lies between 40 and $41\left(T_{\mathrm{m}}\right)$. These strains form a distinct DNA homology group (Hunger \& Claus, 1981).

Source: Soil.

Type strain: DSM 1321 (= NRS 960).

We would like to thank those colleagues listed in Table 1 who kindly provided us with strains, and the SERC for financial support. 


\section{REFERENCES}

Baillie, A. \& Walker, P. D. (1968). Enzymes of thermophilic aerobic spore-forming bacteria. Journal of Applied Bacteriology 31, 114-119.

BATChelor, M. D. (1919). Aerobic, spore-bearing bacteria in the intestinal tract of children. Journal of Bacteriology 4, 23-34.

Berkeley, R. C. W. \& GoOdfellow, M. (editors) (1981). The Aerobic Endospore-forming Bacteria: Classification and Identification. London \& New York: Academic Press.

BoEYÉ, A. \& AERTS, M. (1976). Numerical taxonomy of Bacillus isolates from North Sea sediments. International Journal of Systematic Bacteriology 26, 427441.

Bonde, G. J. (1975). The genus Bacillus. An experiment with cluster analysis. Danish Medical Bulletin 22, 41-61.

BondE, G. J. (1976). The marine Bacillus. Journal of Applied Bacteriology 41, vi-vii.

Castellani, A. (1954). Observations sur quelques ulcérations de la jambe cosmopolites et tropicale: ulcus varicosom et ulcus varicosoides; macroulcus perstans; ulcus tropicum; ulcus veldis, ulcus pyogenicum; ulcus tropicaloides. Minerva dermatologica 29, $1-40$.

Castellani, A. (1955). A brief note on Bacillus cascainensis ( $=$ Krusella cascainensis Cast. 1954), its taxonomic position. Giornale di batteriologia $e$ immunologia 48, 272-273.

Claus, D. \& Berkeley, R. C. W. (1986). Genus Bacillus Cohn 1872. In Bergey's Manual of Systematic Bacteriology, vol. 2, pp. 1105-1140. Edited by P. H. A. Sneath. Baltimore: Williams \& Wilkins.

Cowan, S. T. (1974). Cowan and Steel's Manual for the Identification of Medical Bacteria, 2nd edn. Cambridge: Cambridge University Press.

Delaporte, B. \& Sasson, A. (1967). Étude des bactéries des sols arides du Maroc: Bacillus maroccanus n. sp. Comptes Rendus de l'Academie des Sciences D264, 2344-2346.

Durand, M., Pichinoty, F., Job, C. \& Mandel, M. (1979). Nutrition carbonée et étude taxonomique de Bacillus subtilis et Bacillus licheniformis. Canadian Journal of Microbiology 25, 491-498.

FaHMY, F., Flossdorf, J. \& Claus, D. (1985). The DNA base composition of type strains of the genus Bacillus. Systematic and Applied Microbiology 6, 6065 .

Frazier, N. C. (1926). A method for the detection of changes in gelatin due to bacteria. Journal of Infectious Diseases 39, 302-309.

GiBson, T. (1934). An investigation of the $B$. pasteuri group. I. Description of strains isolated from soils and manures. Journal of Bacteriology 28, 295-311.

GiBson, T. (1935). The urea-decomposing microflora of soils. I. Description and classification of the organisms. Zentralblatt für Bakteriologie, Parasitenkunde, Infektionskrankheiten und Hygiene, Abteilung II 92, 364-380.

Gibson, T. \& GoRdon, R. E. (1974). Bacillus Cohn 1872. In Bergey's Manual of Determinative Bacteriology, 8th edn., pp. 529-550. Edited by R. E. Buchanan \& N. E. Gibbons. Baltimore: Williams \& Wilkins. Gibson, T. \& TopPING, L. E. (1938). Further studies of the aerobic spore-forming bacilli. Proceedings of the Society of Agricultural Bacteriologists 43-44.

GILBERT, R. J. (1979). Bacillus cereus gastroenteritis. In Food-borne Infections and Intoxications, 2 nd edn., pp. 495-518. Edited by H. Riemann \& F. L. Bryan. New York: Academic Press.

Gonzalez, J. M., JR, Brown, B. J. \& Carlton, B. C. (1982). Transfer of Bacillus thuringiensis plasmids coding for $\delta$-endotoxin among strains of $B$. thuringiensis and $B$. cereus. Proceedings of the National Academy of Sciences of the United States of America 79, 6951-6955.

Goodfellow, M. \& CROSs, T. (1984). Classification. In Biology of the Actinomycetes, pp. 7-164. Edited by M. Goodfellow, M. Mordarski \& S. T. Williams. London \& Orlando: Academic Press.

Goodfellow, M. \& Dickinson, C. H. (1985). Delineation and description of microbial populations using numerical methods. In Computer-assisted Bacterial Systematics, pp. 164-226. Edited by M. Goodfellow, D. Jones \& F. G. Priest. Orlando \& London: Academic Press.

Goodfellow, M. \& Williams, E. (1986). New strategies for the selective isolation of industrially important bacteria. Genetic Engineering and Biotechnology Reviews 4, 213-262.

GoRDON, R. E. (1966). Some criteria for the recognition of Nocardia madurae (Vincent) Blanchard. Journal of General Microbiology 45, 355-364.

GoRDON, R. E. (1968). The taxonomy of soil bacteria. In The Ecology of Soil Bacteria, pp. 293-321. Edited by T. R. G. Gray \& D. Parkinson. Liverpool: Liverpool University Press.

GORDON, R. E. (1981). One hundred and seven years of the genus Bacillus. In The Aerobic Endospore-forming Bacteria: Classification and Identification, pp. 1-15. Edited by R. C. W. Berkeley \& M. Goodfellow. London \& New York: Academic Press.

Gordon, R. E., HaYnes, W. C. \& PANG, C. H.-N. (1973). The Genus Bacillus (Agricultural Handbook no. 427). Washington DC: United States Department of Agriculture.

GoRdon, R. E., HYDE, J. L. \& MOORE, J. A., JR (1977) Bacillus firmus-Bacillus lentus: a series or one species? International Journal of Systematic Bacteriology 58, 256-262.

GoTTHEIL, O. (1901). Botanische Beschreibung einiger Bodenbakterien. Zentralblatt für Bakteriologie, Parasitenkunde, Infektionskrankheiten und Hygiene, Abteilung II 7, 680-691.

GyLlenBERG, H. G. \& LAINE, J. J. (1971). Numerical approach to the taxonomy of psychrophilic bacteria. Annales medicinae experimentalis et biologiae fenniae 49, 62-66.

HobBs, G. \& Cross, T. (1983). Identification of endospore-forming bacteria. In The Bacterial Spore, vol. 2, pp. 50-78. Edited by A. Hurst \& G. W. Gould. London: Academic Press.

Hsu, S. C. \& Lockwood, J. L. (1975). Powdered chitin agar as a selective medium for enumeration of actinomycetes in water and soil. Applied Microbiology 29, 422-426.

Hunger, W. \& Claus, D. (1981). Taxonomic studies on Bacillus megaterium and on agarolytic strains. In 
The Aerobic Endospore-forming Bacteria: Classification and Identification, pp. 217-239. Edited by R. C. W. Berkeley \& M. Goodfellow. London \& New York: Academic Press.

KLAUShOFER, H. \& Hollaus, F. (1970). Zur Taxonomie der hochtthermophilen in Zuckerfabrikssaften vorkommenden, aeroben Sporenbildner. Zeitschrift für Zuckerindustrie 20, 465-470.

КосH, A. (1888). Ueber Morphologie und Entwicklungsgeschichte einiger endosporer Bacterienformen. Botanische Zeitung 46, 277-287.

Kramer, J. M., Turnbull, P. C. B., Munshi, G. \& GILBERT, R. J. (1982). Identification and characterization of Bacillus cereus and other Bacillus species associated with foods and food poisoning. In Methods for the Isolation and Identification of Food Poisoning Organisms, pp. 261-268. Edited by J. E. L. Corry, D. Roberts \& F. A. Skinner. London: Academic Press.

KRYCH, V. K., Johnson, J. L. \& Yousten, A. A. (1980). Deoxyribonucleic acid homologies among strains of Bacillus sphaericus. International Journal of Systematic Bacteriology 30, 476-484.

LAINE, J. J. (1970). Studies on psychrotrophic bacteria of food origin. Annales academiae scientiarum fenniae series $A, I V$ biologica $169,1-36$.

LARKIN, J. M. \& StoKes, J. L. (1967). Taxonomy of psychrophilic strains of Bacillus. Journal of Bacteriology 94, 889-895.

Logan, N. \& Berkeley, R. C. W. (1981). Classification and identification of the genus Bacillus using API tests. In The Aerobic Endospore-forming Bacteria: Classification and Identification, pp. 106-140. Edited by R. C. W. Berkeley \& M. Goodfellow. London \& New York: Academic Press.

Logan, N. A., CaPel, B. J., Melling, J. \& Berkeley, R. C. W. (1979). Distinction between emetic and other strains of Bacillus cereus using the API system and numerical methods. FEMS Microbiology Letters 5, 373-375.

MacDonell, M. T. \& Colwell, R. R. (1985). The contribution of numerical taxonomy to the systematics of Gram-negative bacteria. In Computer-assisted Bacterial Systematics, pp. 107-136. Edited by M. Goodfellow, D. Jones \& F. G. Priest. Orlando \& London: Academic Press.

Martin, D. F., Priest, F. G., Todd, C. \& GoodfelLow, M. (1980). Distribution of $\beta$-glucanases within the genus Bacillus. Applied and Environmental Microbiology 40, 1136-1138.

Mishustin, E. N. \& TePper, E. Z. (1948). Description of a new species of bacterium, Bac. longissimus. Microbiologiya 17, 413-414.

Morgan, F., Adams, K. R. \& Priest, F. G. (1979). A cultural method for the detection of pullulandegrading enzymes and its application to the genus Bacillus. Journal of Applied Bacteriology 46, 291-294.

NAKAmURA, L. K. (1984a). Bacillus amylolyticus sp. nov., nom. rev., Bacillus lautus sp. nov., nom. rev., Bacillus pabuli sp. nov., nom. rev. and Bacillus validus sp. nov., nom. rev. International Journal of Systematic Bacteriology 34, 224-226.

NAKAMURA, L. K. (1984b). Bacillus psychrophilus sp. nov. nom. rev. International Journal of Systematic Bacteriology 34, 121-123.

NAKAMURA, L. K. (1987), Deoxyribonucleic acid relatedness of lactose-positive Bacillus subtilis strains and Bacillus amyloliquefaciens. International Journal of Systematic Bacteriology 37, 444-445.

NaKamura, L. K. \& Swezey, J. (1983a). Taxonomy of Bacillus circulans Jordan 1890: base composition and reassociation of deoxyribonucleic acid. International Journal of Systematic Bacteriology 33, 46-52.

Nakamura, L. K. \& Swezey, J. (1983b). Deoxyribonucleic acid relatedness of Bacillus circulans Jordan 1890 strains. International Journal of Systematic Bacteriology 33, 703-708.

NeIDE, E. (1904). Botanische Beschreibung einiger sporenbildenden Bakterien. Zentralblatt für Bakteriologie, Parasitenkunde, Infektionskrankheiten und Hygiene, Abteilung II, 12, 1-32.

O'DONNEll, A. G., NorRIS, J. R., BERKELEY, R. C. W., Claus, D., Kaneko T., Logan, N. A. \& NozaKI, R. (1980). Characterization of Bacillus subtilis, Bacillus pumilus, Bacillus licheniformis and Bacillus amyloliquefaciens by pyrolysis gas-liquid chromatography and by deoxyribonucleic acid (DNA)-DNA hybridization, biochemical tests and API systems. International Journal of Systematic Bacteriology 30, 448-459.

Pichinoty, F. H., DE BaRJaC, H., Mandel, M. \& ASSELINEAU, J. (1983). Description of Bacillus azotoformans sp. nov. International Journal of Systematic Bacteriology 33, 660-662.

Parry, J. M., Turnbull, P. C. B. \& Gibson, J. R. (1983). A Colour Atlas of Bacillus Species. London: Wolfe Medical Publications Ltd.

PRICKETT, P. S. (1928). Thermophilic and thermoduric microorganisms with special reference to species isolated from milk. Descriptions of spore forming types. New York State Agricultural Experiment Station Bulletin 147,1-22.

PRIEST, F. G. (1981). DNA homology in the genus Bacillus. In The Aerobic Endospore-forming Bacteria: Classification and Identification, pp. 33-57. Edited by R. C. W. Berkeley \& M. Goodfellow. London \& New York: Academic Press

Priest, F. G., Goodfellow, M. \& Todd, C. (1981). The genus Bacillus: a numerical analysis. In The Aerobic Endospore-forming Bacteria: Classification and Identification, pp. 91-103. Edited by R. C. W. Berkeley \& M. Goodfellow. London \& New York: Academic Press.

Priest, F. G., Goodfellow, M., Shute, L. A. \& Berkeley, R. C. W. (1987). Bacillus amyloliquefaciens sp. nov. nom. rev. International Journal of Systematic Bacteriology 37, 69-71.

Proom, H. \& KNIGHT, B. C. J. G. (1955). The minimum nutritional requirements of some species in the genus Bacillus. Journal of General Microbiology 13, 474-480.

RUGER, H. J. (1983). Differentiation of Bacillus globisporus, Bacillus marinus comb. nov., Bacillus aminovorans and Bacillus insolitus. International Journal of Systematic Bacteriology 33, 157-161.

SEKI, T., Oshima, T. \& Oshima, Y. (1975). Taxonomic study of Bacillus by deoxyribonucleic acid-deoxyribonucleic acid hybridization and interspecific transformation. International Journal of Systematic Bacteriology 25, 258-270.

Seki, T., Chung, C.-K., Mikami, H. \& Oshima, Y. (1978). Deoxyribonucleic acid homology and taxon- 
omy of the genus Bacillus. International Journal of Systematic Bacteriology 28, 182-189.

SeKI, T., Minoda, M., Yagi, J.-I. \& Oshima, H. Y. (1983). Deoxyribonucleic acid reassociation between strains of Bacillus firmus, Bacillus lentus and intermediate strains. International Journal of Systematic Bacteriology 33, 401-403.

Sharp, R. J., Bown, K. J. \& Atkinson, A. (1980). Phenotypic and genotypic characterization of some thermophilic species of Bacillus. Journal of General Microbiology 117, 201-210.

SiERra, G. (1957). A simple method for the detection of lipolytic activity of microorganisms and some observations on the influence of the contact between cells and fatty substrates. Antonie van Leeuwenhoek 23, 15-22.

Skerman, V. B. D., McGowan, V. \& Sneath, P. H. A. (1980). Approved lists of bacterial names. International Journal of Systematic Bacteriology 30, 225-420.

SMith, N. R., GoRDON, R. E. \& ClaRK, F. E. (1946). Aerobic Mesophilic Sporeforming Bacteria (United States Department of Agriculture Miscellaneous Publication no. 559). Washington, DC: USDA.

Smith, N. R., Gordon, R. E. \& Clark, F. E. (1952). Aerobic Sporeforming Bacteria (United States Department of Agriculture Monograph no. 16). Washington, DC: USDA.

SNEATH, P. H. A. \& Johnson, R. (1972). The influence on numerical taxonomic similarities of errors in microbiological tests. Journal of General Microbiology 72, 377-392.

SNEATH, P. H. A. \& SoKal, R. R. (1973). Numerical Taxonomy. The Principles and Practice of Numerical Classification. San Francisco: W. H. Freeman.

SOMERVILLE, H. J. \& JONES, M. L. (1972). DNA competition experiments within the Bacillus cereus group of bacilli. Journal of General Microbiology 73, 257-261.

StackebrandT, E. \& Woese, C. R. (1981). The evolution of prokaryotes. In Molecular and Cellular Aspects of Microbial Evolution, pp. 1-31. Edited by M. J. Carlile, J. F. Collins \& B. E. B. Moseley. London: Academic Press

StackebrandT, E., LudWig, W., Weizenegger, M., Dorn, S., MCGill, T. J., FoX, G. E., Woese, C. R., SCHUBERT, W. \& SchleIfFER, K.-M. (1987). Comparative 16S rRNA oligonucleotide analyses and murein types of round-spore-forming bacilli and non-spore-forming relatives. Journal of General Microbiology 133, 2523-2529.

Turnbull, P. C. B., Jørgensen, K., Kramer, J. M., GILBERT, R. J. \& PARRY, J. M. (1979). Severe clinical conditions associated with Bacillus cereus and the apparent involvement of exotoxins. Journal of Clinical Pathology 32, 289-293.

WALKER, P. D. \& Wolf, J. (1971). Taxonomy of Bacillus stearothermophilus. In Spore Research 1971, pp. 247-262. Edited by A. N. Barker, G. W. Gould \& J. Wolf. London: Academic Press.

WeLKer, N. E. \& CAMPBELL, L. L. (1967). Unrelatedness of Bacillus amyloliquefaciens and Bacillus subtilis. Journal of Bacteriology 94, 1124-1130.

WERNER, W. (1933). Botanische Beschreibung haufiger am Buttersaureablau beteiligter sporenbildner Baktierspezies. Zentralblatt für Bakteriologie, Parasitenkunde, Infectionskrankheiten und Hygiene, Abteilung II 87, 446-475.

Williams, S. T., Goodfellow, M., Alderson, G., Wellington, E. M. H., Sneath, P. H. A. \& Sackin, M. J. (1983). Numerical classification of Streptomyces and related genera. Journal of General Microbiology 129, 1743-1813.

Williams, S. T., Vickers, J. C. \& Goodfellow, M. (1985). Application of new theoretical concepts to the identification of streptomyces. In Computerassisted Bacterial Systematics, pp. 289-306. Edited by M. Goodfellow, D. Jones \& F. G. Priest. London: Academic Press.

WISHART, D. (1978). Clustan User Manual. Version IC, Release 2, 3rd edn. Edinburgh: Edinburgh University Program Unit.

WOLF, J. \& BARKER, A. N. (1968). The genus Bacillus: aids to the identification of its species. In Identification Methods for Microbiologists, Part B, pp. 93-109. Edited by M. Gibb \& D. A. Shapton. London: Academic Press.

Wolf, J. Chowdbury, M. S. U. (1971). Taxonomy of $B$. circulans and B. stearothermophilus. In Spore Research 1971, pp. 349-350. Edited by A. N. Barker, G. W. Gould \& J. Wolf. London: Academic Press.

Wolf, J. \& SharP, R. (1981). Taxonomic and related aspects of thermophiles within the genus Bacillus. In The Aerobic Endospore-forming Bacteria: Classification and Identification, pp. 251-296. Edited by R. C. W. Berkeley \& M. Goodfellow. London \& New York: Academic Press. 